\title{
EDITORIAL AND REVIEW
}

\section{Theoretical Chemistry: Molecular Spectroscopy and Dynamics} 39th Symposium on Theoretical Chemistry 2003 (STC 2003)
28 September to 2 October 2003, Gwatt, Lake Thun, Switzerland

\author{
Fabio Mariottia ${ }^{a}$ Martin Quack ${ }^{a \star}$, Martin Willeke ${ }^{a}$, and Jürgen Stohner ${ }^{b}$
}

\begin{abstract}
We provide a short review of the scientific meeting defined by the title of this article, which may at the same time serve as a compact review of the field with a substantial number of references to recent work. Exciting new developments in experiments on high-resolution molecular spectra and their analysis as well as new theoretical developments in the calculations of such spectra and the related time-independent and time-dependent quantum dynamics of molecules have led to new answers but also to new questions in the fields of molecular kinetics, molecular reaction dynamics, molecular chaos and statistical mechanics as well as fundamental symmetries in molecular processes. Particular stress is placed on fundamental aspects and new directions.
\end{abstract}

Keywords: Chemical reaction kinetics · Molecular dynamics · Molecular spectroscopy · Quantum chemistry · Theoretical chemistry

$\overline{{ }^{*} \text { Correspondence: Prof. M. Quack }}$

aphysical Chemistry

ETH Zürich (Hönggerberg)

$\mathrm{CH}-8093$ Zürich

Tel.: +411632 4421

Fax: +4116321021

E-Mail: Martin@Quack.ch

http://www.ir.ethz.ch/

bInst. of Chemistry and Biological Chemistry

ZHW Winterthur

$\mathrm{CH}$ 8401-Winterthur

\# A Symposium under the Auspices of the Division Chemical Research of the Swiss Chemical Society (SCS) and the Arbeitsgemeinschaft Theoretische Chemie (DBG, DPG, GDCh) with Conference Chairmen Hans Peter Lüthi and Martin Quack (ETH Zürich) and Jürgen Stohner (Zürich University of Applied Sciences Winterthur)

\section{Introduction}

The present Special Issue of CHIMIA is devoted to the scientific subject as mentioned in the title of this review and which was also the topic of a scientific meeting in Gwatt, on the shore of Lake Thun in the fall of 2003. The 39th Symposium on Theoretical Chemistry (STC 2003) was the latest in an annual series of meetings with a long tradition in Europe and in Switzerland. Indeed, it was the tenth time that the meeting returned to Switzerland with the following previous times, locations, and chairmen: 1966 Zürich (Heinrich Labhart), 1969 Spiez (Georges Wagnière), 1972 Genève (Laurens Jansen), 1976 Basel (Martin Jungen), 1980 Wildhaus (Klaus Müller), 1984 Emmetten (Ulrich Müller-Herold), 1988 Pontresina (Karl Lendi), 1994 Fiesch (Hanspeter Huber), 1998 Gwatt (Walter Thiel). This time, the meeting was organized by Hans-Peter Lüthi and Martin Quack from the Swiss Federal Institute of Technology (ETH) Zürich and Jürgen Stohner from the Zürich University of Applied Sciences Winterthur (ZHW) under the Auspices of the Division Chemical Research (DCR) of the Swiss Chemical Society (SCS) and the
Arbeitsgemeinschaft Theoretische Chemie (AGTC) of the Deutsche Bunsengesellschaft (DBG), the Deutsche Physikalische Gesellschaft (DPG), and the Gesellschaft Deutscher Chemiker (GDCh). About 170 participants attended the symposium which offered 28 lectures of $45 \mathrm{~min}$ each and two poster sessions with a total of about 125 posters. The main theme of the meeting STC 2003 is at the heart of the interactions of experiment and theory in chemistry, as the location was in the heart of Switzerland, near the capital and the Bernese mountains (indeed, the Abstract Book included some basic history of Switzerland [1]). Molecular Spectroscopy has always had a special relation to Theoretical Chemistry, and the theme of the meeting follows an earlier one about a decade ago [2]. In the present short review we introduce this special issue of CHIMIA by summarizing the lectures and some of the discussions of the meeting with fairly extensive references, and thus this may also serve as a very compact review of the current status of at least part of the field. This is then complemented by a small selection of papers from some of the invited speakers. It was not possible here to include 
more for reasons of space and cost, but we hope that our review provides at least some insight into those aspects not represented in the following papers. These include at least contributions from several groups at Swiss universities as well as one from Germany and two from France. Given the theme of the meeting, there is an exceptional proportion of experimental work presented in such a theoretical meeting, which led to many stimulating interactions.

\section{Session 1}

After a short welcome by the organizers and the chairman (Martin Quack) of Session 1, the meeting started already Sunday evening with an opening lecture by Jürgen Troe, University of Göttingen (Fig. 1) on 'Reaction Dynamics with Wavepackets, Adiabatic Channels, and Classical Trajectories'. Whereas quantum scattering calculations of inelastic collisions are most accurate, the numerical effort is prohibitive for larger systems involving many atoms. Capture rate constants dominate chemical kinetics of barrierless reactions and statistical theories, such as the statistical adiabatic channel model, have proven useful in this context [3-5]. It has been demonstrated recently that the combination of classical trajectory calculations (with full quantum treatment of the capture) with adiabatic channel calculations describe capture rate constants even down to very low temperatures (milli Kelvin) where quantum effects are expected to be most prominent [6-9]. Ingredients for the calculation of capture rate constants are potential energy surfaces, including for example ion-dipole interactions for charged reactants $\left(e . g . \mathrm{O}_{2}{ }^{+}+\right.$

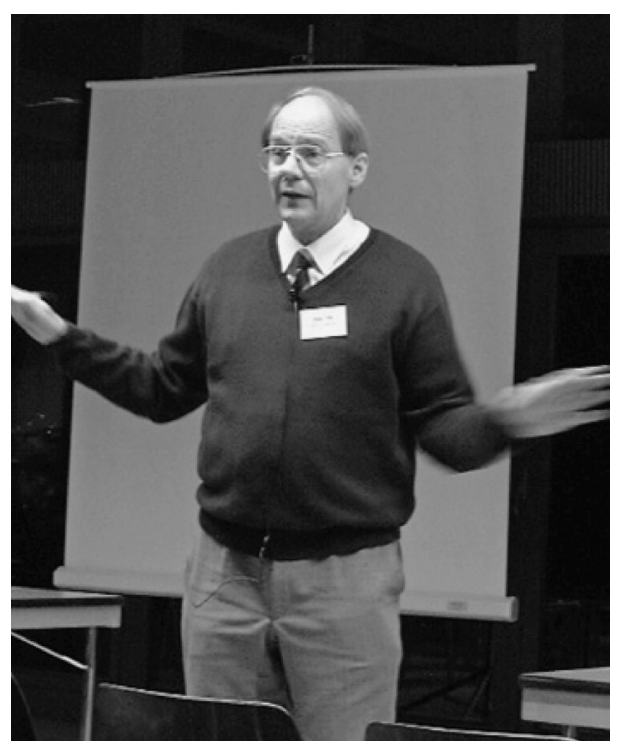

Fig. 1. Jürgen Troe, Göttingen, explaining reactions lively with both hands, starting to make a high flight in his lecture
$\mathrm{C}_{8} \mathrm{H}_{10}$ ), permanent dipole and quadrupole potentials, and adiabatic channels including centrifugal potentials. Jürgen Troe discussed also certain reactions of great importance for the chemistry of the atmosphere such as $\mathrm{H}+\mathrm{O}_{2}$ (and isotopomeric versions with $\mathrm{D}, \mathrm{T}, \mathrm{Mu}$ ) and $\mathrm{OH}+\mathrm{OH}$. In the latter case, a global analytical potential energy hypersurface exists [10], and the speaker pointed to the need for such potential energy hypersurfaces for dynamical calculations. Beyond the technical aspects, he also provided the audience with a lively journey through time and history, mentioning his early associations with the following speaker (Martin Jungen), the chairman and some of the older members in the audience, such as Tino Gäumann, who had not missed the opportunity to join this event.

Martin Jungen, University of Basel (Fig. 2) talked on 'What is a Muon Doing in Atoms or Molecules?'. The muon $\mu$ is an elementary particle belonging to the Lepton family. Its mass is about 207 times the mass of the electron or about $11 \%$ of the atomic mass unit $(\approx 0.11 \mathrm{u})$. It has a mean lifetime of about $2.2 \times 10^{-6} \mathrm{~s}$. It decays into an electron or positron plus neutrinos and exists as particle and anti-particle $\left(\mu^{+}\right.$and $\left.\mu^{-}\right) . \mu^{+}$can be considered as a light proton, whereas $\mu^{-}$ could be termed a heavy electron. $\left(\mu^{+} e^{-}\right)$ can be considered a light isotope of hydrogen (Muonium, $\mathrm{Mu}$ ). In a theoretical study, 'exotic' molecules have been investigated which include one $\mu^{-}$particle: $\left(\mathrm{pp} \mu^{-}\right)^{+}$ (which would correspond to $\mathrm{H}_{2}^{+}$), $\left(\alpha \alpha \mu^{-} e^{-}\right)^{2+}$ (corresponding to $\mathrm{He}_{2}{ }^{2+}$ ). Martin Jungen discussed various aspects of bonding in these exotic systems as well as the role of the Born-Oppenheimer approximation [11]. He also mentioned the potential importance of $\left(\mathrm{p}^{+} \mathrm{p}^{+} \mu^{-}\right)^{+}$for myonic fusion.

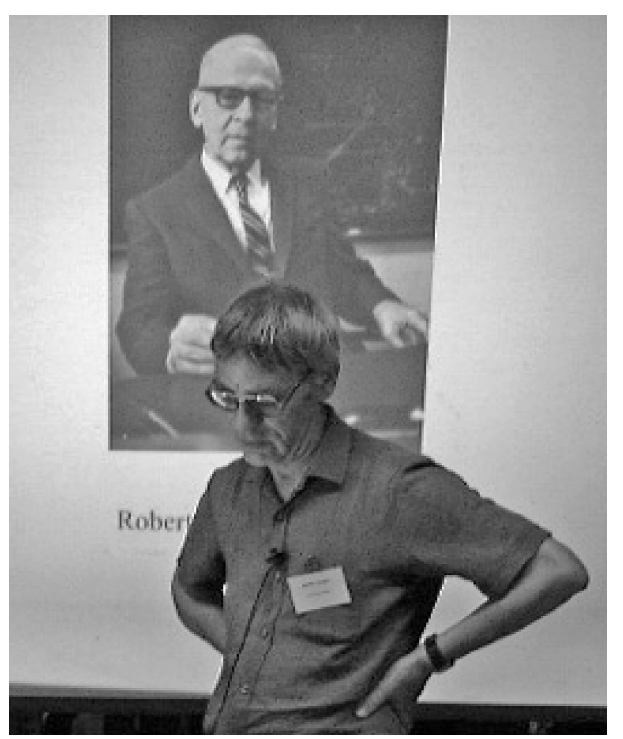

Fig. 2. Martin Jungen, Basel, with a picture of Robert Mulliken in the background, from whose famous lecture the title of Martin Jungen's lecture was adapted

\section{Session 2}

This session chaired by Werner Kutzelnigg, University of Bochum (Fig. 3) started on Monday with a talk given by Wim Klopper, University of Karlsruhe (Fig. 4) on 'Explicitly-Correlated Calculations of Excitation Energies'. The background of these techniques, which owe much to the chairman and the speaker himself in this session, was lucidly introduced. Explicitly-correlated coupled cluster calculations up to triple excitations are almost routine in high-quality $a b$ initio studies of potential energy hypersurfaces, energetic and spectroscopic quantities for molecules with less than six atoms [12]. The computational accuracy reached demands to consider various corrections (e.g. relativistic spin-orbit and non Born-Oppenheimer corrections). Very large one-electron basis sets are needed (with high orbital angular

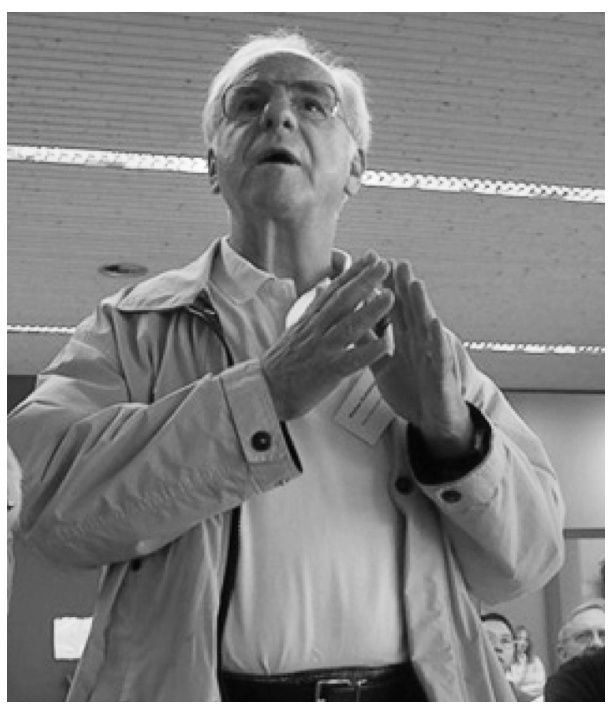

Fig. 3. Werner Kutzelnigg, Bochum

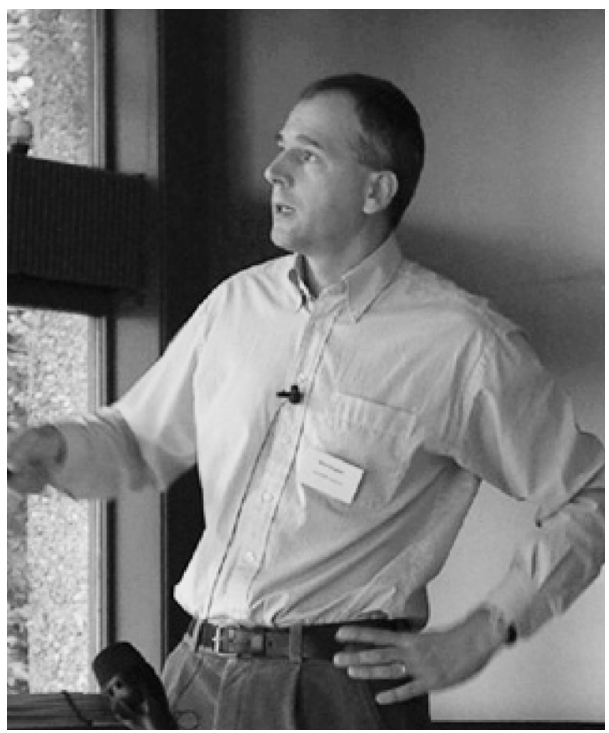

Fig. 4. Wim Klopper, Karlsruhe, in his clear and highly instructive lecture 
momentum quantum number $l$ ) which are costly or even computationally prohibitive, simplified treatments are strongly needed to treat even larger molecules. $\mathrm{CC} 2(\mathrm{R} 12)$ and $\mathrm{CCSD}(\mathrm{R} 12)$ are suitable methods for larger molecules [13]. The R12 method describes the Coulomb hole and the electron-cusp correctly by introducing as a scaling factor the inter-electron distance. However, this factor does not vanish for large electronic distances; with a Gaussian damping factor for the scaling efficient computational techniques with linear scaling can be used [14]. It has been demonstrated that excitation energies converge faster with $\mathrm{CC} 2$ than with $\operatorname{CCSD}(\mathrm{T})$. Although excitation energies of small molecules are close to experiment [12], CC2(R12) has sometimes difficulties with the symmetry of the excited state.

David Luckhaus, University of Göttingen (Fig. 5) reported on 'Direct MultiArrangement Quantum Dynamics: From Dynamics to Reaction' by presenting full six-dimensional quantum dynamics results on HONO overtone spectroscopy and reaction dynamics [15-17].

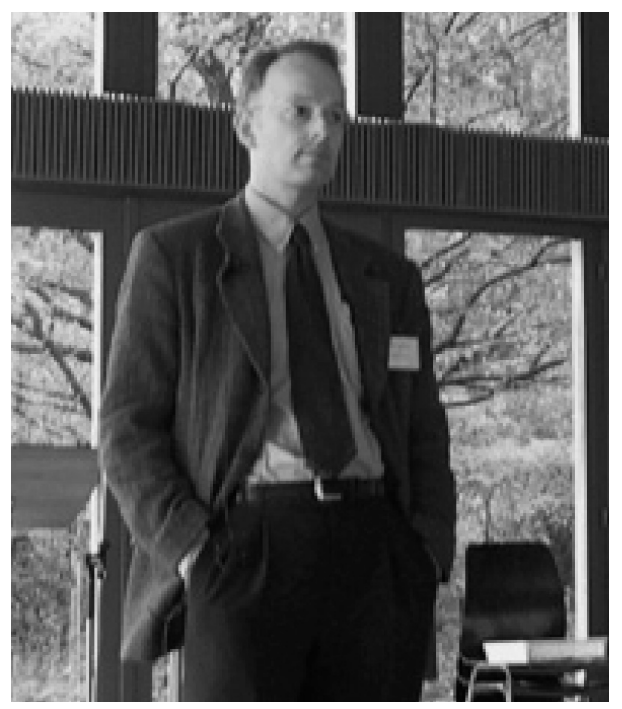

Fig. 5. David Luckhaus, Göttingen

A multi-dimensional potential energy (PES) and dipole moment hypersurface has been obtained using density functional theory (B3LYP functional, 6-311++G** basis set) in a 'direct' way without analytically fitting the $a b$ initio data points. The data points have been interpolated using 'successively averaged spline interpolation' (SASI) to a desired grid size. Convergence of this procedure has carefully been checked. Luckhaus investigated the cis-trans isomerization as well as the hydrogen shift based on 6D variational calculations of overtone spectra and 6D wavepacket dynamics. Potential-optimized discrete variable representation (PODVR) combined with adiabatic contraction has been used to obtain the $\mathrm{OH}$ overtone spectrum up to six quanta of $\mathrm{OH}$-stretching excitation (about $22000 \mathrm{~cm}^{-1}$ ) for the two stable HONO isomers. Numerous perturbations have been identified and analyzed. In combination with band intensities and intensity distributions this allows a thorough understanding of intramolecular dynamics up to very high vibrational excitations well into the visible spectral region. He presented a new multi-arrangement formulation of the dynamics in local valence coordinates which allows to describe tunneling in full dimensionality at very high excitation. This provides a detailed picture of the quantum dynamics for cisHONO trans-HONO isomerization and of 1,3-hydrogen transfer reactions.

\section{Session 3}

Session 3 was the first session devoted mostly to experiments and was chaired by Peter Botschwina, University of Göttingen, who introduced John P. Maier, University of Basel (Fig. 6) speaking about 'Electronic Spectra of Carbon Chains: Relevance to Astrophysics and Nanoscience'.

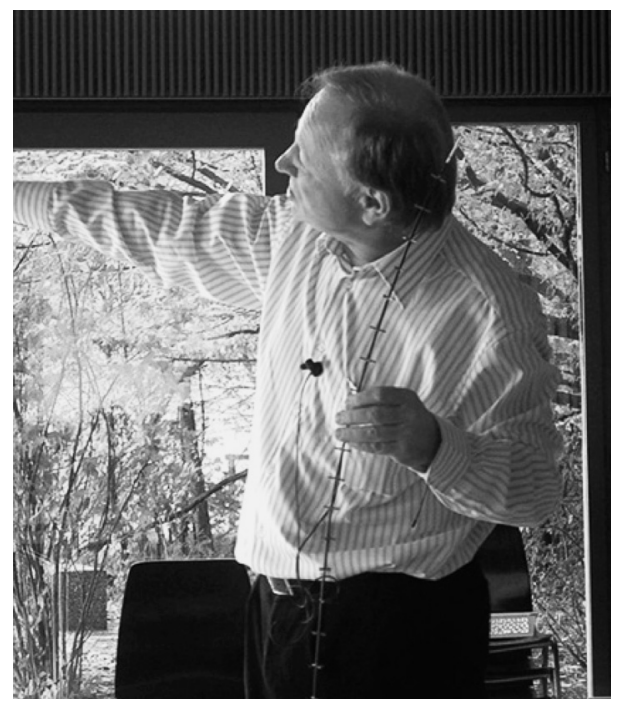

Fig. 6. John P. Maier, Basel, with a model of a long carbon chain molecule in one hand

Carbon chain radicals like $\mathrm{C}_{n} \mathrm{H}$ $(2 \leq \mathrm{n} \leq 8)$ can be found in dark interstellar clouds and 'odd' chains of the type $\mathrm{HC}_{2 \mathrm{n}+1} \mathrm{H}$ are believed to exist in the interstellar medium. These 'odd' chains are much more reactive than the 'even' chains $\mathrm{HC}_{2 \mathrm{n}} \mathrm{H}$. The various compounds mentioned are relevant for an understanding of the chemistry in interstellar medium. Very sensitive spectroscopic techniques (cavity ring-down spectroscopy with supersonic jet expansions [18][19], recently extended by the Basel group to treat ions [20], as well as frequency modulation absorption spectroscopy) are needed to examine those compounds in the laboratory which then al- lows to identify carbon chains through their electronic absorption spectrum. The odd chains are more reactive and shortlived and are investigated by mass-selective resonant two-photon ionization techniques (R2C2PI, [21]). Understanding the electronic spectra poses a challenge to theory since one would require line positions to better than $1 \mathrm{~cm}^{-1}$ for the carbon chain absorptions between 400 and $800 \mathrm{~nm}$. Such accuracy can hardly be reached by pure $a b$ initio theory to date for such molecules. Thus, laboratory spectroscopy must help out at present. Another important quantity is the oscillator strength which together with accurate line positions would facilitate the assignment of electronic spectra in dark molecular clouds (temperature about $10 \mathrm{~K}, 10^{4}$ particles per $\mathrm{cm}^{3}$ ) and diffuse interstellar clouds (temperature about $100 \mathrm{~K}$, $10^{2}$ particles per $\mathrm{cm}^{3}$ ). An investigation of electronic transitions $\mathrm{A}^{1} \Delta_{u} \leftarrow \mathrm{X}^{1} \Sigma^{+}{ }_{g}$ and B ${ }^{1} \Sigma^{+}{ }_{u} \leftarrow \mathrm{X}^{1} \Sigma^{+}{ }_{g}$ as a function of chain length $4 \leq n \leq 7\left(\mathrm{HC}_{2 \mathrm{n}}{ }_{\mathrm{H}}^{\mathrm{H}}\right)$ shows that the A-B state gap is far from zero (which would be expected for a one-dimensional crystal) even for the rather long $\mathrm{HC}_{14} \mathrm{H}$ chain (gap of about $2 \mathrm{eV}$ ). Those investigations may also be used to test or calibrate models of molecular electronic devices [22].

Frederic Merkt, ETH Zürich (Fig. 7) reported on 'High-Resolution VUV Spectroscopy, Rydberg States and Cations' with rare gas dimers $\left(\mathrm{Ar}_{2}^{+}\right)$as examples. Potential energy functions for six electronically excited states have been derived from highresolution photoelectron spectroscopy with an accuracy of 0.06 to $2 \mathrm{~cm}^{-1}$ [23]. F. Merkt then pointed to new developments from his group allowing for high resolution of 0.008 $\mathrm{cm}^{-1}$ in the VUV. The derived potential energy functions agree reasonably well with those calculated $a b$ initio. Accurate measurements of the hyperfine structure in ${ }^{83} \mathrm{Kr}$

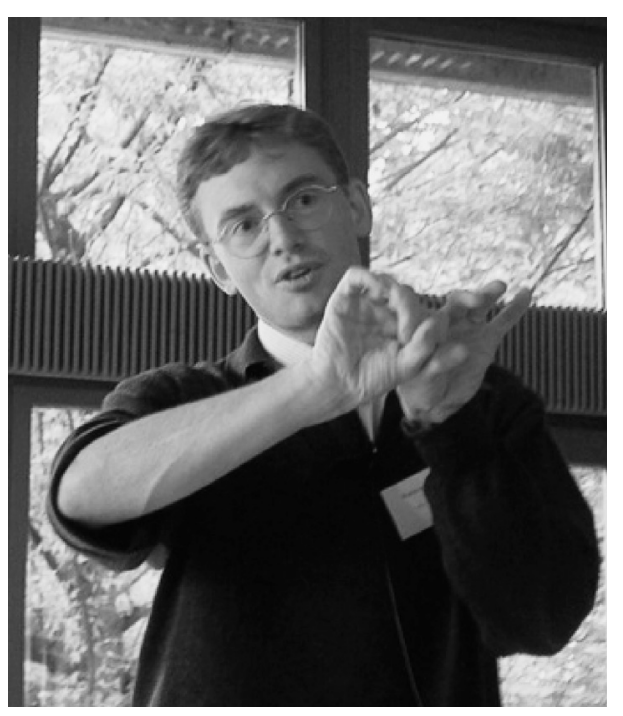

Fig. 7. Frederic Merkt, Zürich, explaining some of the special properties of Rydberg states 
by pulsed-field ionization techniques ( $n$ s and $n$ d Rydberg states with the principal quantum number $n$ ranging from 30 to 190) revealed nuclear spin dependence of the appearance of the Rydberg state spectra, especially at high $n$, that leads to hyperfine splittings. Multichannel quantum defect theory (MQDT) is adequate to explain the observed features [24]. A more complete account of his lecture and related work appears in this issue of CHIMIA [25].

\section{Session 4}

This session was in turn devoted to theory with Wilfried Meyer (Fig. 8, University of Kaiserslautern) as chairman and started with a talk by Hans-Joachim Werner, University of Stuttgart (Fig. 9) on 'ExplicitlyCorrelated Total Wavefunctions: LMP2R12 with Density Fitting'. High-level elec-

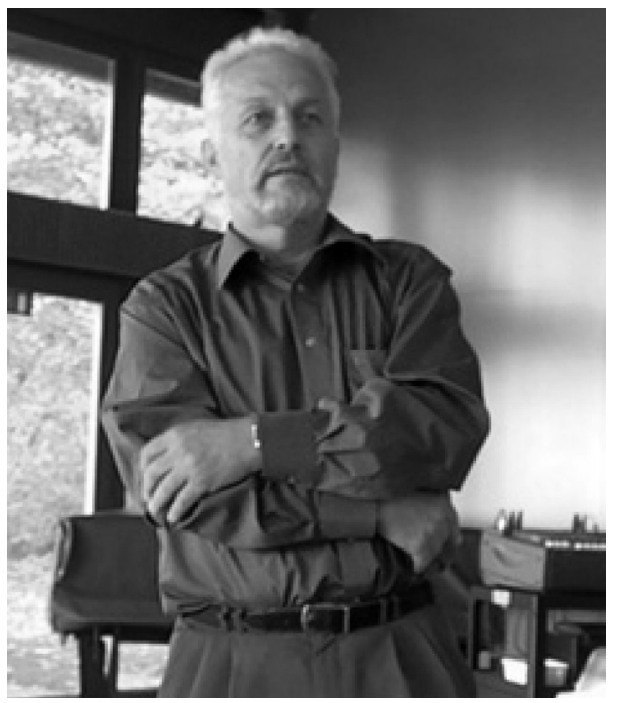

Fig. 8. Wilfried Meyer, Kaiserslautern, as chairman

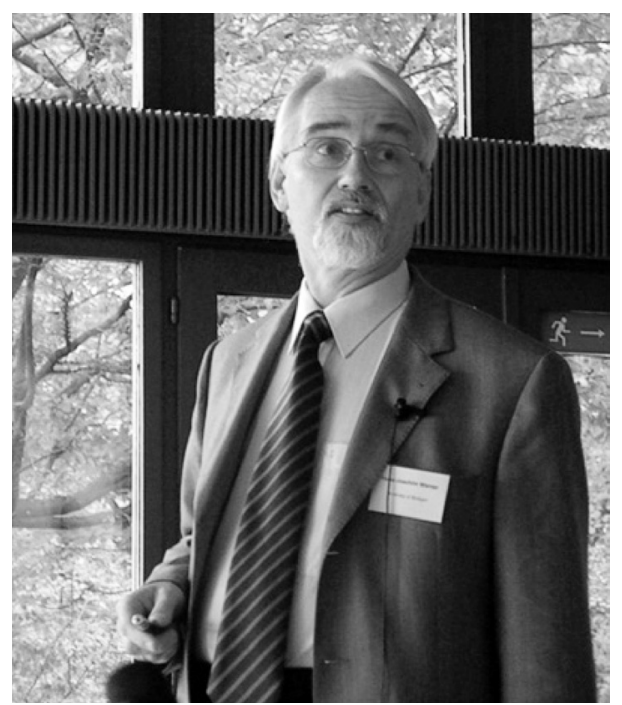

Fig. 9. Hans-Joachim Werner, Stuttgart, giving a thoughtful reply to a question tronic structure calculations suffer from strongly non-linear scaling with system size and with size of the atomic basis set. The calculation of 2-electron integrals and their transformation is a true bottleneck. Although electron correlation is important for high-quality calculations, it converges rather slowly with basis set size (orbital angular momentum quantum number $l$ ). Local density fitting (LDF) procedures strongly enhance convergence.

LDF-MP2 [26] and LDF-CCSD [27] scale linearly with molecular size. A costly part of the calculation is the four-index integral evaluation due to its unfavorable scaling with respect to atomic basis set size. Density fitting is able to change this scaling considerably by introducing individual excitation subspaces (domains; their size is independent of the size of the molecular system) for electron pairs and a multipole expansion for the generation of the 2-electron integrals for distant pairs. Different fitting bases for each electron pair can result in scaling down to $N$ as compared to $N^{3}$ (with $N$ the atomic orbital basis set size). The optimization of methanol pentamer and t-butanol pentamer structures is feasible and LMP2 is probably faster than DFT. A LDF-MP2 combined with R12 is most desirable.

Jeppe Olsen, University of Aarhus (Fig. 10) reported on 'Coupled Cluster Expansions with Quadruple and Higher Excitations' to calculate accurate thermochemical and spectroscopic data, in particular atomization energies and harmonic vibrational frequencies [28][29]. With increasing quality from $\operatorname{CCSD}(\mathrm{T})$ (perturbative triple excitations), CCSDT, CCSDTQ (quadruple excitations) to CCSDTQ5 (quintuple excitations), atomization energies of diatomics can be calculated with an accuracy of about $0.5 \mathrm{~kJ} / \mathrm{mol}$ (in case of $\mathrm{N}_{2}$ with CCSDTQ5). Olsen discussed a number of difficult cases such as $\mathrm{F}_{2}, \mathrm{C}_{2} \mathrm{H}_{4}$, HOF and $\mathrm{O}_{3}$. It is important to include all electrons in the correlation treatment as well as using core valence basis sets, which, however, need to be very large (up to quintuple-zeta quality). Small errors in the atomization energies obtained with $\operatorname{CCSD}(\mathrm{T})$ are fortuitous due to error cancellation. The outstanding lecture of Jeppe Olsen actually stimulated some discussion on how to best compare very accurate $a b$ initio results with spectroscopic experiments, a question of obvious interest in general and for the mixed audience represented here in particular. For instance, one way would be to compare indirectly obtained 'experimental' $r_{e}$-structures with the directly obtained $a b$ initio equilibrium geometry. Another possibility would be to calculate theoretical transition frequencies using a full potential and rovibrational dynamics, a routine method for diatomic molecules, but difficult for polyatomic mole- cules. Also the data set would become very large and not transparent in the case of polyatomic molecules. An intermediate procedure would be to do full dimensional (or high dimensional) calculations on appropriate potential energy hypersurfaces for line frequencies and fit these using spectroscopic effective Hamiltonians with reduced parameter sets. This may perhaps be the most meaningful approach, which is certainly feasible for diatomic molecules and it was also pointed out that such procedures were already used for polyatomic molecules [10][30-36] even though sometimes with accuracy limited by the multidimensional Quantum Monte Carlo technique or by reduced dimensionality treatments. Further discussion of this important matter followed later in the lectures by $\mathrm{K}$. Hirao, W. Thiel, and J. Gauss.

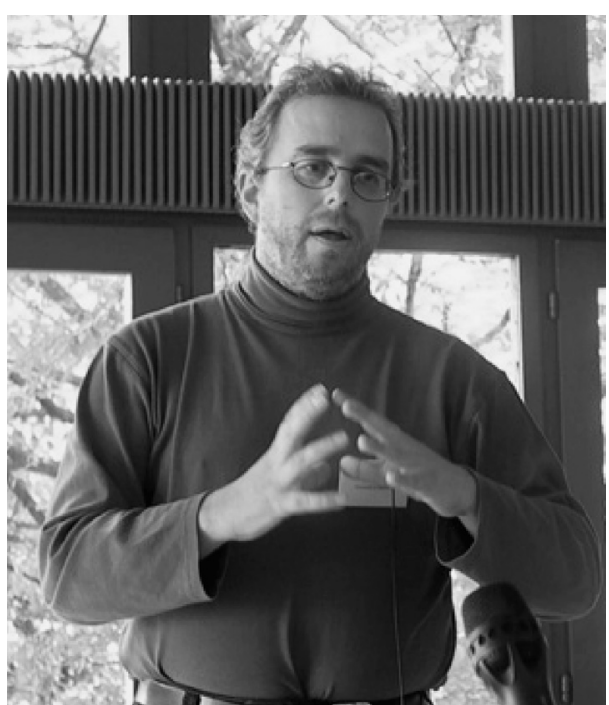

Fig. 10. Jeppe Olsen, Aarhus, explaining the difficulties of highly accurate calculations

\section{Session 5}

The next session was chaired by Jürgen Hinze, University of Bielefeld (Fig. 11) and the opening talk was given by Gernot Frenking, University of Marburg (Fig. 12) entitled 'The Nature of the Chemical Bond - Old Questions, New Answers'. He discussed the ability of molecular orbital (MO) and valence bond (VB) models to rationalize the physical origin of the chemical bond in relation to heuristic bonding models as presented in chemistry textbooks. However, as Frenking pointed out, these often neglect knowledge on the physical origin of the chemical bond gained in theoretical studies by Hellmann, Hirshfeld, Ruedenberg, Kutzelnigg, Schwarz and others. The basis of his work goes back to the energy decomposition schemes proposed by Ziegler [37] and Morokuma [38] in the 70s. Frenking reviewed the Energy Partitioning Analysis (EPA) addressing a correct partitioning of the energy in order to describe 


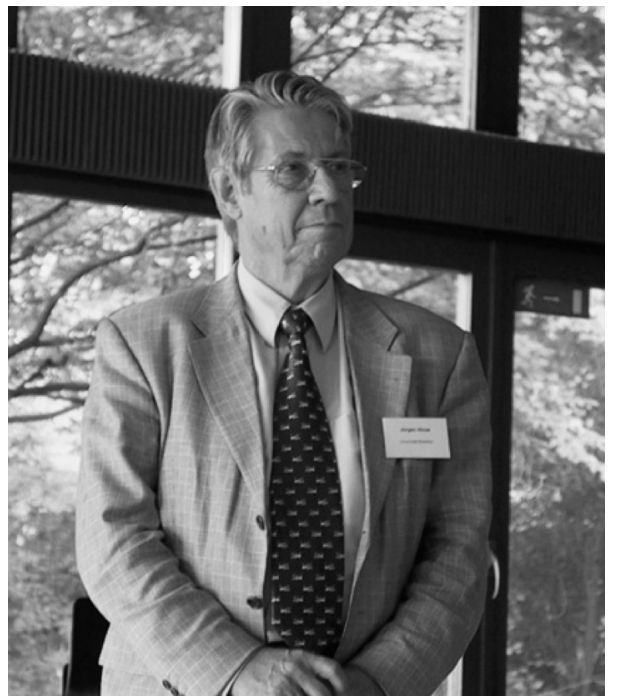

Fig. 11. Jürgen Hinze, Bielefeld, one of the senior chairmen of the field and this meeting

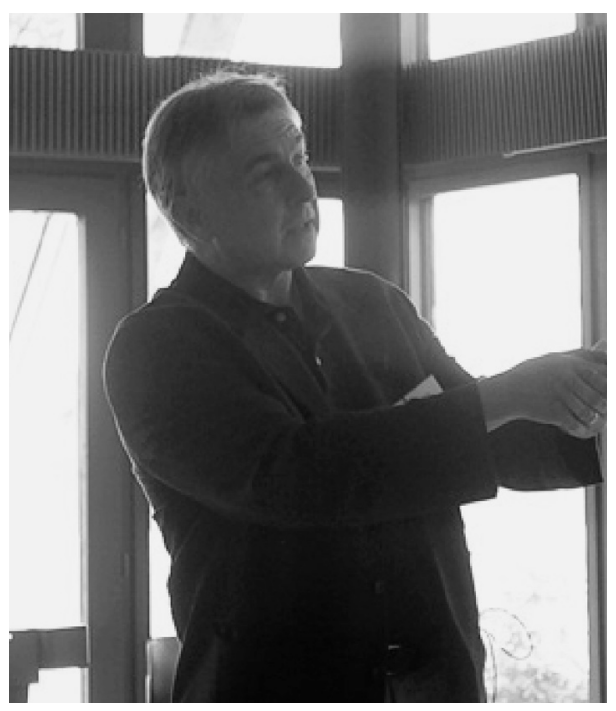

Fig. 12. Gernot Frenking, Marburg

the electrostatic or covalent nature of the bond. Particular attention was given to the role of electrostatic attraction and Pauli repulsion which, following Frenking's ideas, have to be considered separately, these being two well-defined terms with a clear physical meaning. Besides the brief introduction to the approach he presented a consistent number of examples, mainly metal complexes and molecules of main group element systems, where new answers were given. A recent discussion of these subjects from Frenking and coworkers can be found in [39] and [40]. The chemical bond being central to theoretical chemistry a long discussion followed Frenking's presentation.

Kimihiko Hirao, University of Tokyo (Fig. 13) held the PCCP lecture sponsored by the journal Phys. Chem. Chem. Phys. The title of his lecture was 'Recent Advances in Electronic Structure Theory'. Third-order Douglas-Kroll (DK3) coupled cluster methods have been used up to con- nected quadruple substitutions. Hirao reported spectroscopic quantities like rotational constants (accurate to about 0.02 $\mathrm{cm}^{-1}$ ), vibration-rotation interaction constants (accurate to about $0.01 \mathrm{~cm}^{-1}$ ), harmonic vibrational frequencies (accurate to about $8 \mathrm{~cm}^{-1}$ ), and dissociation energies (accurate to about $0.02 \mathrm{eV}$ ) for hydrides of the second to the fifth row of the periodic table [41]. DK3 results are almost comparable to solutions obtained by solving the Dirac equation. Furthermore, the relative stability of three $\mathrm{SiC}_{3}$ isomers has been determined by second-order perturbation theory with multiconfigurational self-consistent field reference functions (GMC-PT). The results obtained by this method confirm the earlier relative stability as reported by Fritz Schaefer's group [42].

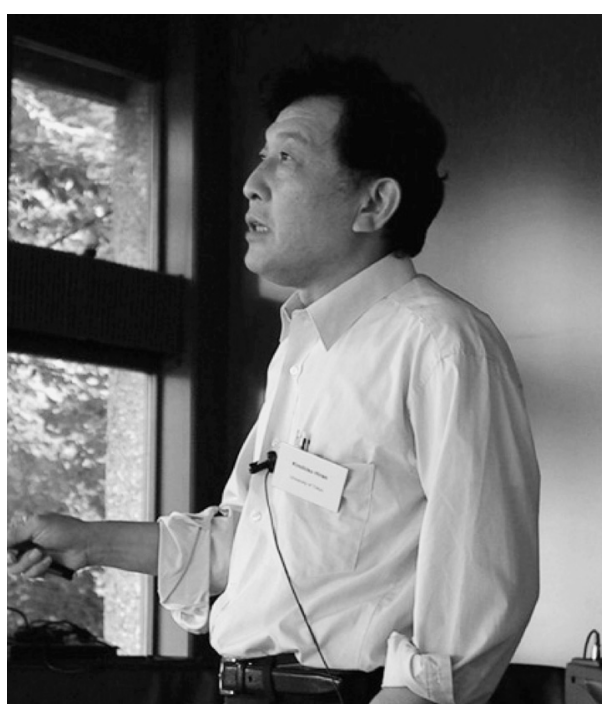

Fig. 13. Kimihiko Hirao, Tokyo, the PCCP lecturer of this meeting

\section{Session 6}

The Tuesday morning's Session 6 started with the Hellmann Prize award ceremony chaired by Walter Thiel (MPI Mülheim) as chairman of the Arbeitsgemeinschaft für Theoretische Chemie (AGTC). Traditionally, the name of the prize winner is kept confidential until the award ceremony and he appears as speaker N.N. in the conference program. Only some participants might have noticed that, while an abstract for the lecture N.N. did not appear among the lecture abstracts at the beginning of the abstract book, there was one abstract on page 145 in the later collection of contributed papers where the footnote 'poster' was missing, indicating either a misprint - or else that this was the missing Hellmann Prize lecture. Indeed, Georg Kresse, University of Vienna (Fig. 14) received this prize for his contributions to developing and efficiently implementing plane wave density functional methods allowing simulations of solids and solid surfaces (see homepage of

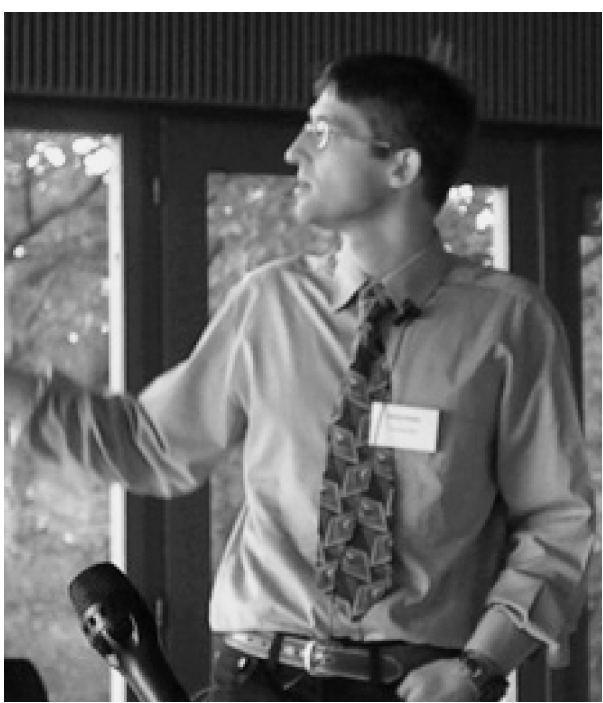

Fig. 14. Georg Kresse, Vienna, this year's Hellmann Prize winner

the AGTC at http://www.agtc.uni-bonn.de and [43]).

In his lecture on the 'Importance of Single-Electron Energies for the Description of CO Adsorption on Surfaces' he discussed the failure of semilocal density functionals in properly describing the stable adsorption site of $\mathrm{CO}$ on $\mathrm{Pt}(111)$. This failure is rationalized by investigating the interaction of the highest occupied molecular orbital (HOMO) and the lowest unoccupied molecular orbital (LUMO) of CO with the platinum surface and it points to a deficiency in the present local and semilocal functionals which leads to an underestimation of the HOMO/LUMO gap. Functionals which are corrected for self interaction or which include Hartree-Fock exchange seem to better describe the experimental findings [44][45]. This lecture as well stimulated quite some discussion on various density functional approaches, as also on the possible need for treating nuclear motion as well by quantum dynamics.

The following lecture by Rainer $D$. Beck, EPF Lausanne (Fig. 15) dealt with experiments on molecule surface interactions [47][46]. He addressed the "Vibrational Mode-Specific Reaction of Methane on a Nickel Surface'. Beck reported experiments on state-resolved sticking coefficients for vibrationally excited $\mathrm{CH}_{4}$ on a single crystal nickel surface. The methane chemisorption reaction is a prototype for the activated dissociative chemisorption of a polyatomic molecule. The experimental setup involves the generation of a molecular beam of methane prepared in specific vibrationally excited states and the determination of the chemisorption products after the impact with the metal surface using Auger electron spectroscopy. With these tools the Lausanne group contributed to the debate on whether the chemisorption reaction of $\mathrm{CH}_{4}$ on a $\mathrm{Ni}(100)$ surface is statisti- 


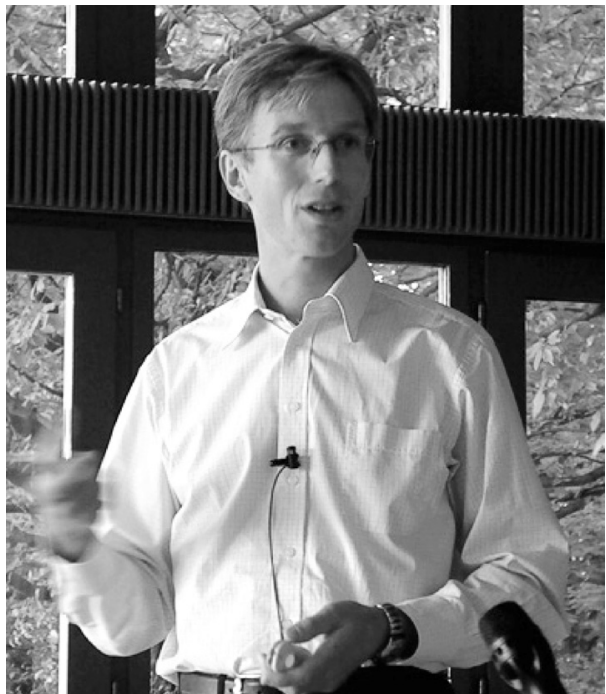

Fig. 15. Rainer D. Beck, Lausanne

cal or mode-specific. It has been shown that the latter is indeed the case as it was found in the gas-phase reactivity of methane. A more complete account of this work is provided in the present issue [46].

\section{Session 7}

Session 7, which was chaired by HansPeter Lüthi, ETH Zürich, was opened by a lecture by Roberto Marquardt, University of Marne-La-Vallee (Fig. 16) entitled 'Vibrational and Rotational Wave Packet Motion in Polyatomic Molecular Systems' and included actually both the theory of gasphase isolated molecule dynamics and gas-surface interactions. Within the spectroscopic approach for the calculation of the time-dependent quantum dynamics in polyatomic molecules he discussed how to determine global potential energy and dipole moment surfaces. Being a prototype for chemical reactions and adsorption

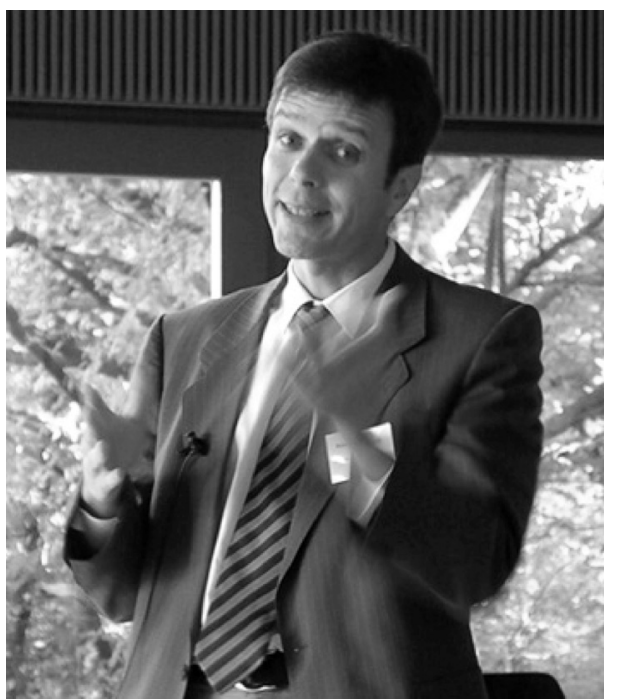

Fig. 16. Roberto Marquardt, Marne-La-Vallee processes, the laser-assisted inversion of ammonia was the main subject of his lecture. With the help of a detailed investigation of the vibrational wave packet motion in a four-dimensional treatment of the timedependent excitations and intra-molecular dynamics, he analyzed the roles of the various mechanisms for the inversion motion of highly vibrationally excited ammonia molecules. In particular he showed that even at energies well above the inversion barrier, the dominating process is still tunneling when compared with the less competitive intra-molecular vibrational redistribution and direct excitation [48]. The basic components of these studies are the introduction of the concept of the infrared $\mathrm{NH}$ chromophore as the group of NH stretching and bending vibrations in NHXY compounds which in turn allows for the definition of a 4-dimensional subspace and the calculation of vibrational eigenfunctions and eigenvalues within this 4D space using Watson's Hamiltonian. Details on a global electric dipole function of ammonia can be found in [49]. The last part of the talk was dedicated to the role of rotations and molecular orientation. The lecture included as well some very thoughtful comments on the construction of potential hypersurfaces for gas molecule-surface interactions and a more complete account can be found in this issue [50].

Quantum computing was one of the main subjects of Regina de Vivie-Riedle's (Universität München, Fig. 17) talk. While 'quantum algorithms' have been already published only few 'qubit machine' implementations are available at a laboratory level. In this talk we heard about new physical implementations which involve vibrationally excited molecules. Beside computing and information storage devices the approach involved different tools from the theory of spectroscopy and dynamics of molecules. In first place we are in the range of ultrafast molecular processes [52] which are also of interest in chemical or photophysical reactions dynamics [34][51]. Control of these processes is then required and can be reached using coherent laser light and specially designed laser pulse shapes. De Vivie-Riedle showed how a basic control tool (the 'Optimal Control Theory' (OCT) [53][54]) can be extended and used to find the optimal laser field to manipulate complex molecules. Regina de VivieRiedle presented data and examples for a new quantum computer implementation. The main quantum chemical data were extracted from HF, CIS, DFT and TDDFT calculations which are suited for large molecular frames and allow for a setup of complex embedded systems (see for example the Wesolowski talk). Regina de VivieRiedle also discussed electrocyclic reactions as molecular switches, where in the discussion the point was brought up that chiral molecules had been calculated for their use as femtoseconds switches [55]. Altogether the work presented opens avenues to an interesting research field.

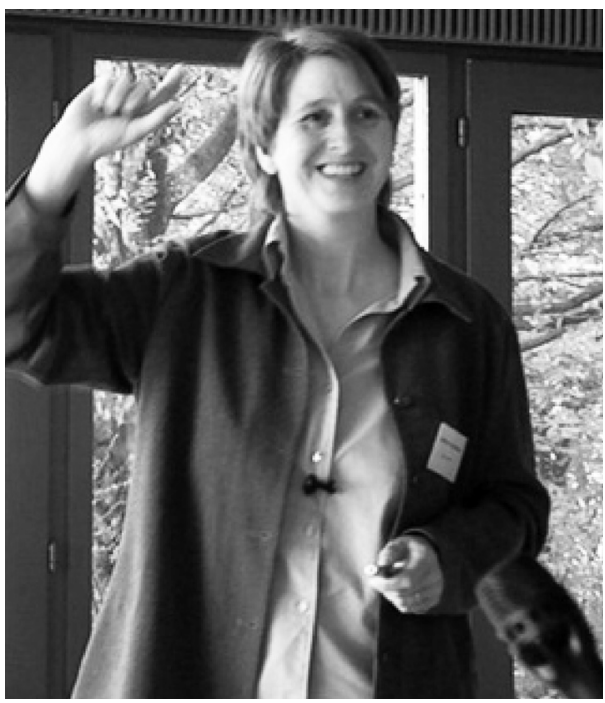

Fig. 17. Regina de Vivie-Riedle, München. In a fresh and smiling presentation.

\section{Session 8}

Dirk Schwarzer, University of Göttingen (Fig. 18) was Chairman of Session 8. Samuel Leutwyler's (University of Berne, Fig. 19) lecture on an experimental topic touched on another fundamental subject: Hydrogen bonds which play an important role in biology and chemistry [56]. He showed that in a 7-hydroxyquinoline $\left(\mathrm{NH}_{3}\right)_{3}$ cluster $\left(7 \mathrm{HQ} \cdot\left(\mathrm{NH}_{3}\right)_{3}\right)$ a single $\mathrm{H}$ atom transfer can be induced along the ammonia wire. In particular after initiation via $S_{1} \leftarrow S_{0}$ excitation the reaction does not proceed directly from the vibrational ground state of the $S_{1}$ state but additional excitations are required to activate the $\mathrm{H}$ transfer reaction. CASSCF with a 6-

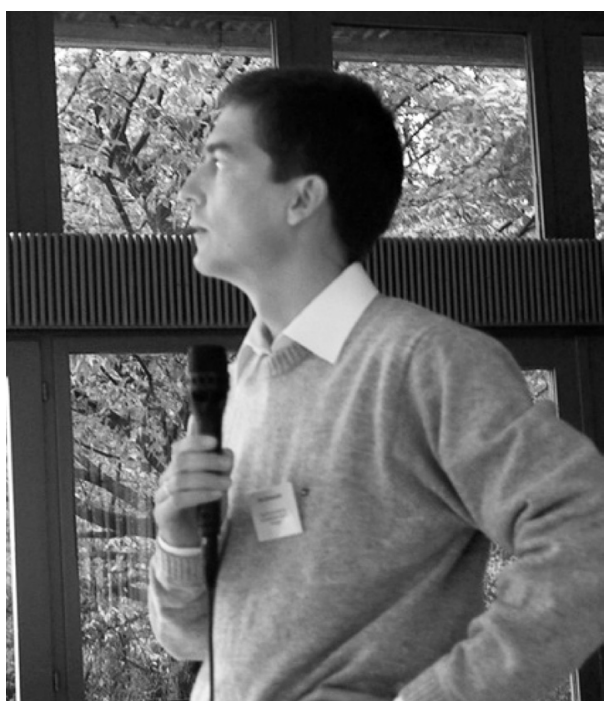

Fig. 18. Dirk Schwarzer, Göttingen 


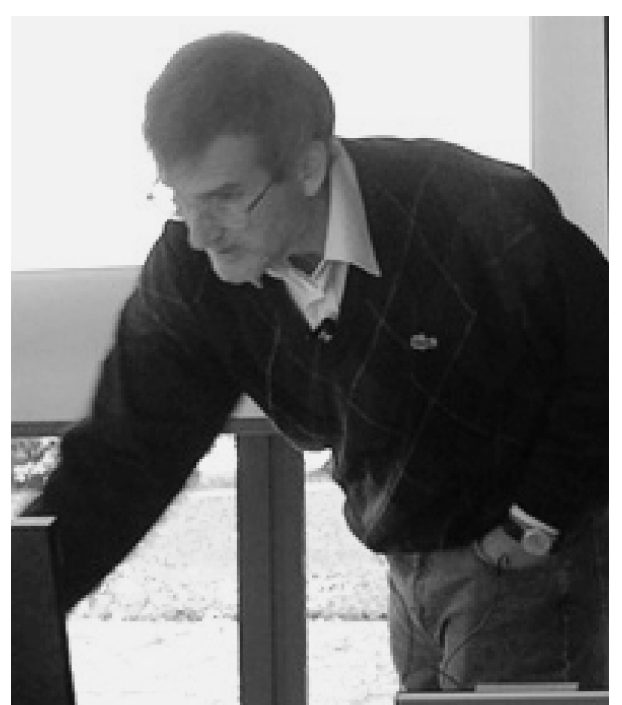

Fig. 19. Samuel Leutwyler, Bern

$31(+) \mathrm{G}(d, p)$ basis set was used to rationalize and predict experimental data obtained performing laser UV analysis after having synthesized and cooled the 7HQ. $\left(\mathrm{NH}_{3}\right)_{3}$ clusters in a $20 \mathrm{~Hz}$ pulsed supersonic jet expansion of $\mathrm{Ne}$ mixed with $1 \% \mathrm{NH}_{3}$. A more detailed report on this subject can be found in [57] and in this issue a summary report appears as well [58].

Hydrogen bonding is also one of the research subjects of Martina Havenith, Ruhr-Universität Bochum (Fig. 20) who reported on two spectroscopic measurements: a gas-phase disaccharide and the fully analyzed gas-phase high-resolution IR spectrum of the formic acid dimer. There is experimental evidence for double proton transfer tunneling in formic acid dimer [59] which is a prototype for double hydrogen bonded organic complexes. Havenith's group fully resolved the tunneling splitting and determined a ground-state tunneling frequency of $86 \mathrm{MHz}$ and a C-O vibrationally excited state tunneling-frequency of $300 \mathrm{MHz}$. The experimental procedure

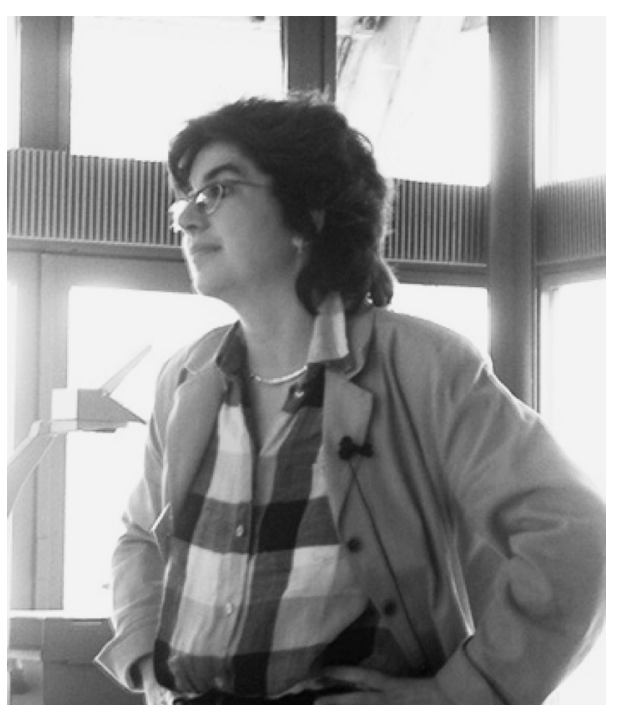

Fig. 20. Martina Havenith, Bochum involves the formation of the clusters in a supersonic jet expansion and spectroscopic measurements using a lead salt diode laser. Beside the importance of these data for biological studies she underlined the lack of reliable theoretical models for these systems. There was some discussion on the unsuccessful search for imaginary frequencies in the alleged transition state and the existence of an open formic acid dimer with only one hydrogen bond, postulated before on the basis of kinetic and spectroscopic evidence [60][61].

\section{Session 9}

As chairman of Session 9, Eugen $H$. Schwarz, University of Siegen (Fig. 21) introduced Marius Lewerenz. University of Paris (Fig. 22) who continued his series of studies on hydrogen bonds. In particular he presented IR spectroscopic results for the hydrogen-bonded dimers thiirane-HF and thiirane-DF whose analysis has revealed a global anharmonic coupling between all low frequency intermolecular modes and the high frequency HF/DF stretching mode. He pointed out that commonly used theoretical models might not be sufficient for the calculation of the red shift induced by the formation of the hydrogen bond and he presented results from an anharmonic and multidimensional theoretical treatment of the vibrational dynamics obtained over a part of a complete $a b$ initio potential energy surface. In the second part of his talk, M. Lewerenz addressed very weakly bound van der Waals complexes. The target system was the $\left(\mathrm{NeHe}_{\mathrm{n}}\right)^{+}$cluster class as example for a successful application of Quantum Monte Carlo sampling combined with a diatomics in molecules (DIM) approach. The calculations reveal the existence of a stable symmetric triatomic ion core $(\mathrm{He}-\mathrm{Ne}-\mathrm{He})^{+}$. A detailed summary of this

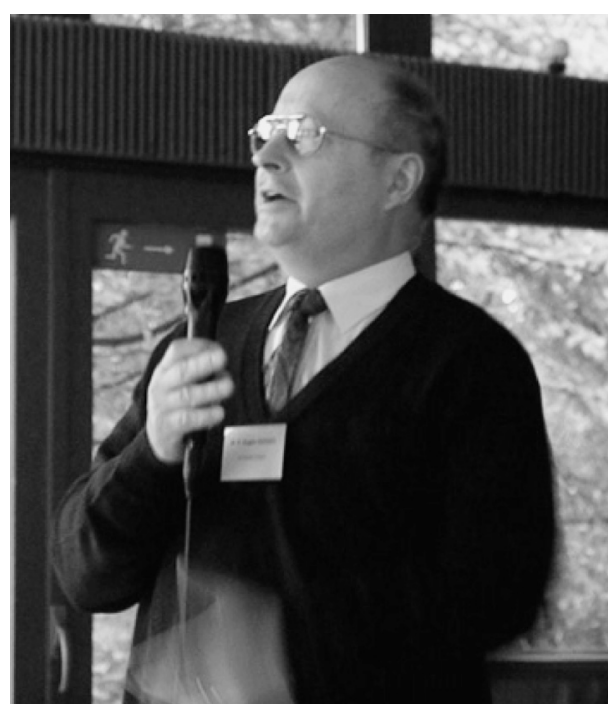

Fig. 21. Eugen H. Schwarz, Siegen

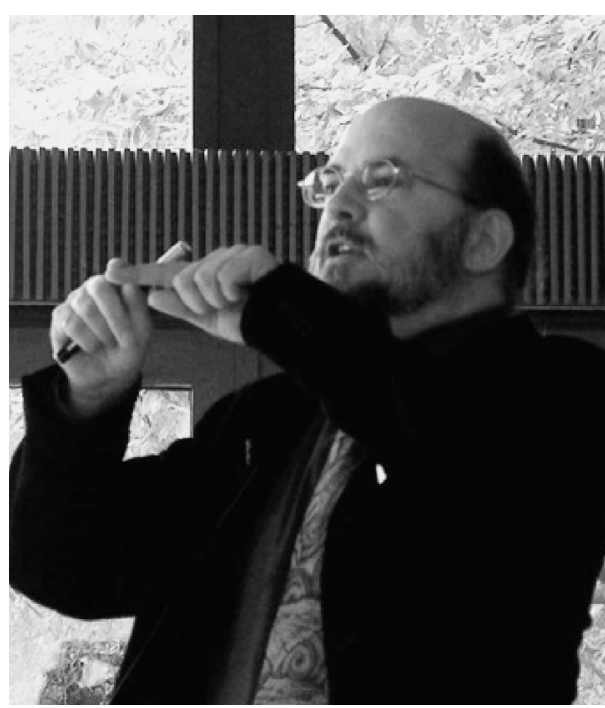

Fig. 22. Marius Lewerenz, Paris

work can be found in the present issue of CHIMIA [62].

Walter Thiel, MPI Mülheim (Fig. 23) reviewed the state of the art in the theoretical approaches to obtain spectroscopic parameters from $a b$ initio calculations. The key step of the method includes the computation of harmonic and anharmonic force fields followed by a rovibrational perturbation treatment. He presented the application of this approach to a series of small reactive molecules and discussed the results with particular attention to the convergence of the theoretical methods [63]. The second half of the talk addressed variational calculation on ammonia molecule [64] which was repeatedly a topic at the meeting [48-50][65]. The method chosen for the calculation of the PES was coupled cluster $\operatorname{CCSD}(\mathrm{T})$ with extrapolation to the complete basis set. Thiel showed that it is possible to systematically obtain results just a few $\mathrm{cm}^{-1}$ away from experimental data if appropriate tools are used. He underlined that corrections (for example to account for

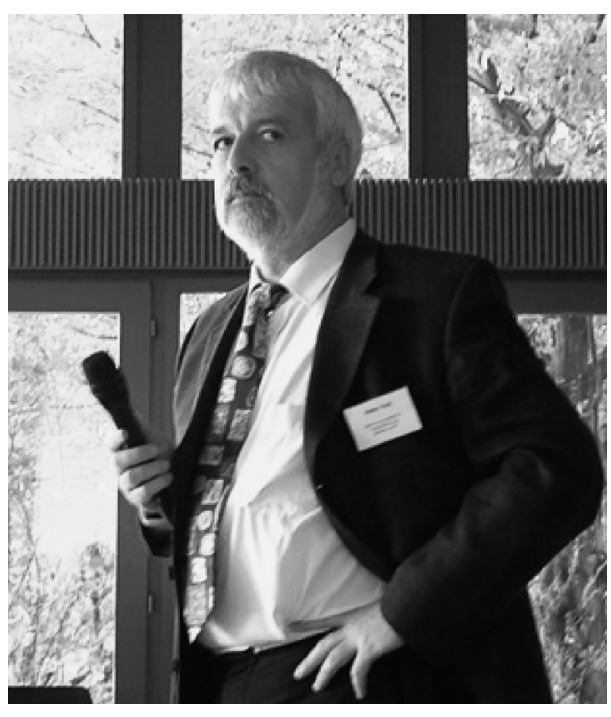

Fig. 23. Walter Thiel, Mülheim 
relativistic effects) are required and that particular attention should be paid to the accuracy of the calculation at every single step of the procedure. A more complete summary of this work can be found in this issue [66].

\section{Session 10}

The Chairman of Session 10 was Frédéric Merkt, ETH Zürich (Fig. 7). The image of handshaking molecules was appropriately given by Martin Suhm, University of Göttingen (Fig. 24) in his talk about chiral recognition and self-recognition. Chiral recognition is well known to play a key role in biochemical processes and organic synthesis and Suhm explored this field with a systematic study which involved the combination of theoretical and experimental spectroscopic techniques. The presented chemical systems are for example glycidol [67], closely related to the important oxirane-carbonitrile [68] which has been studied for biochemical selection in evolution and parity violation, and lactates [69] where the chiral self-recognition (i.e. the ability of a molecule to distinguish between a copy or a mirror copy of itself) arises from intermolecular interactions mainly due to hydrogen bonds. Weak secondary interactions, involving for example $\mathrm{CH}-\mathrm{O}$, were also the subject of interest for the determination of chiral discrimination. The main experimental data are obtained via Ragout-jet FTIR spectroscopy (a rapidscan FTIR supersonic jet technique) that has evolved to a very efficient technique over the years [70][71]. The experimental results were rationalized with quantum chemistry calculations [72][73].

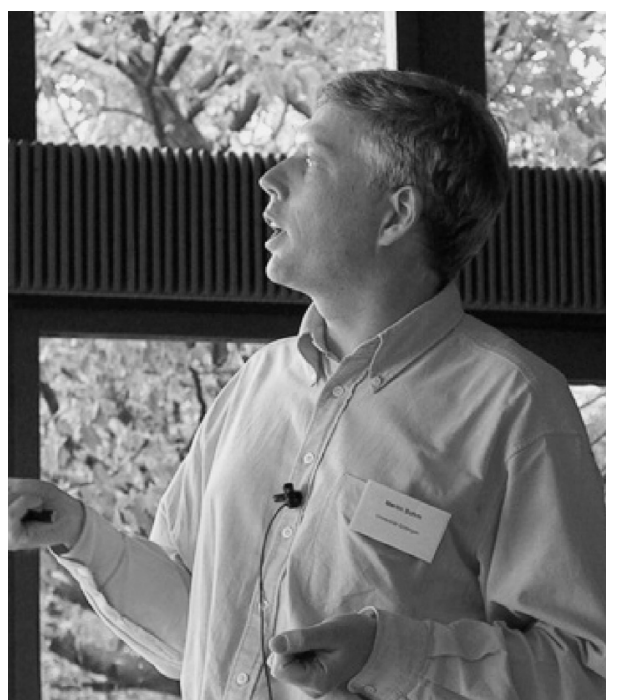

Fig. 24. Martin Suhm, Göttingen, with left and right hands in almost symmetric positions
Tomasz A. Wesolowski, University of Geneva (Fig. 25) gave a lecture entitled 'Electron Density Partitioning as a Route Towards Accurate Multi-Scale Modeling of Complex Molecular Systems and Materials'. He presented the recent theoretical developments and applications of a density functional theory approach, which is based on the so-called total energy bi-functional $E\left[\rho_{A}, \rho_{B}\right]$ already used in early work by Gordon and Kim and developed to a powerful tool by Wesolowski and his group for computer modeling of complex polyatomic systems at quantum mechanical level. $\mathrm{He}$ explained that in this approach the effective potential coupling of two subsystems is expressed in the orbital-free embedding formalism [74][75]. This formalism allows the

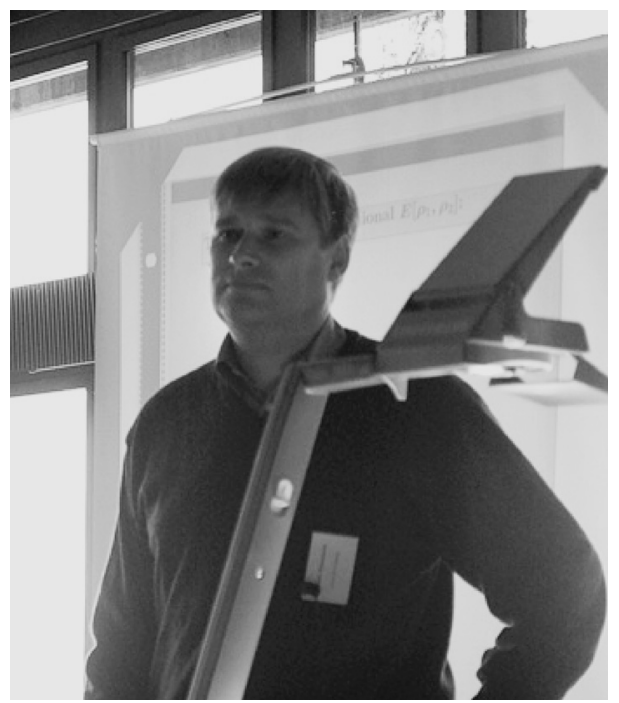

Fig. 25. Tomasz A. Wesolowski, Geneva

calculation of electronic-structure-dependent properties of an embedded subsystem without the construction of the wavefunction of the whole system comprising the molecule under investigation and its microscopic environment (for example an atom in a solid, molecule in a solvent, molecule in a porous material or molecule on a surface). Then he also reviewed some challenging issues of density functional theory (DFT) such as approximating the kinetic energy functional or describing weak intermolecular interactions (e.g. Ne-Ne, Ar-Ar). He noted that these are of particular significance for practical applications of the orbital-free embedding formalism. In the last part of his lecture he mentioned a recent extension of this formalism, which allows even the study of localized excited states in a condensed phase. A more detailed summary appears in the present issue [76].

\section{Session 11}

This session was chaired by Kim Baldrige, University of Zürich (Fig. 26), who introduced Joachim Sauer, Humboldt-Universität, Berlin (Fig. 27) giving a lecture with the title 'Calculations on Transition Metal Oxides -From Gas Phase Clusters to Solid Catalysts'. In the first part he talked about theoretical investigations of vanadium oxides as selective catalyst for partial oxidations. Vanadium oxides are particularly interesting because their reactivities show a dependence on the aggregation level [77][78]. As model systems a thin film of $\mathrm{V}_{2} \mathrm{O}_{5}$ on e.g. $\alpha-\mathrm{Al}_{2} \mathrm{O}_{3}$ or $\mathrm{SiO}_{2}$ is used. He presented calculations of the energy profile for the selective oxidation of methanol to formaldehyde [79][80]. This reaction has the advantage that it is well investigated experimentally with solid vanadium oxide as a catalyst as well as in the gas phase for the related molecular cation (with mass spectrometry). The corresponding calculations show large differences in reactivity between cationic gas phase species and neutral clusters and support the experimental results. He discussed the reliability

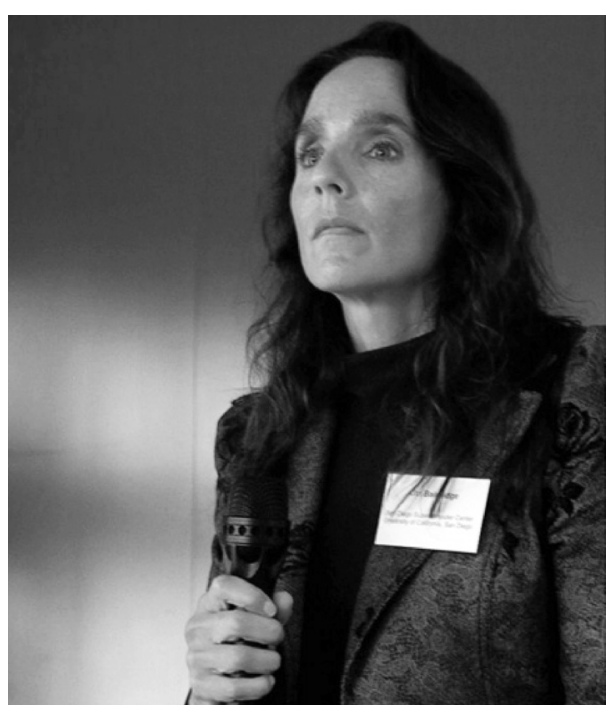

Fig. 26. Kim Baldrige, Zürich University

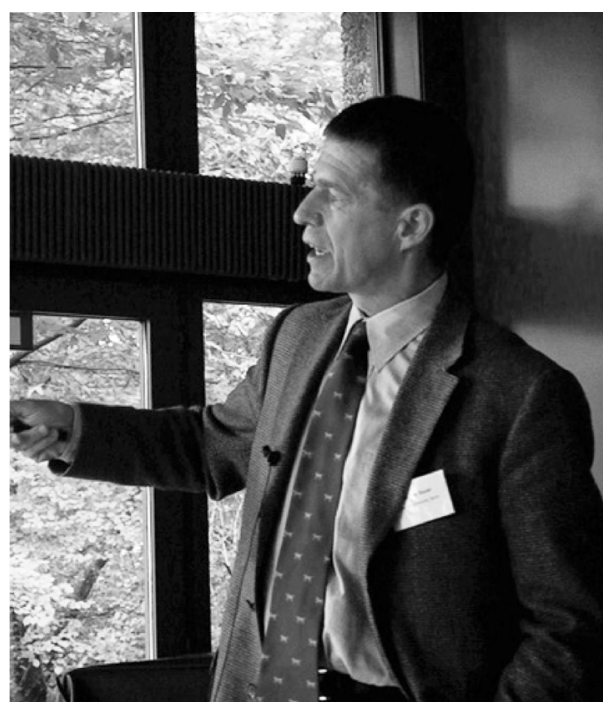

Fig. 27. Joachim Sauer, Humboldt University Berlin 
of DFT for the description of the oxygen-metal bond and the activation barrier. He mentioned that the methanol oxidation is one of the rare examples where DFT calculations (with PW91 or B3LYP functionals) yield too-high reaction barriers. Some evidence was found that this is caused by the biradical character of the transition state for this system [81].

This day of the conference closed with a lecture by Claude A. Daul, University of Fribourg (Fig. 28) entitled 'Modeling Magnetic and Spectroscopical Properties of Coordination Compounds'. He reported recent results on a new DFT-based ligand field model and its application to study magnetic and spectroscopic properties of coordination compounds including investigations of his group on a first principle study of the nephelauxetic effect. They compared Racah's parameter A and B for the free metal ions with the corresponding values in a

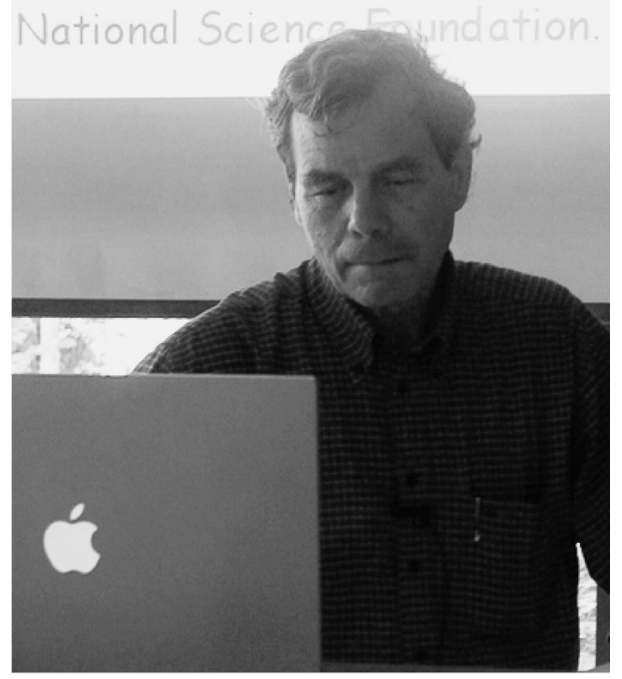

Fig. 28. Claude A. Daul, Fribourg

crystal field. He mentioned that in such investigations the covalent reduction increases with increasing transition metal oxidation state in the series $\mathrm{CrO}_{4}{ }^{4-}, \mathrm{Mn} \mathrm{O}_{4}{ }^{4-}$, $\mathrm{FeO}_{4}{ }^{4-}$ and with an increasing metal-ligand bond covalency in the series $\mathrm{CrX}_{4}(\mathrm{X}=\mathrm{F}$, $\mathrm{Cl}, \mathrm{Br}, \mathrm{I})$. He further showed that this approach can also be utilized for magnetic exchange coupling in homonuclear transition metal dimer complexes. This approach is based on a model of localized d-electrons and on a procedure allowing to express its parameters in terms of exchange and Coulomb integrals from DFT calculations of a homonuclear dimer complex. He described how it is possible to relate these integrals with the manifold of all Slater determinants of the active space originating from the magnetic electrons of the dimer. This method has been successfully applied to $\mathrm{d}(9)$ and $\mathrm{d}(1)$ model clusters involving $\mathrm{Cu}^{\mathrm{II}}$ and $\mathrm{Ti}_{2} \mathrm{Cl}_{9}{ }^{3-}$. He further mentioned that his group was also able to extend this approach to cases with more than one unpaired electron per site. A more detailed summary of this research can be found in the present issue of CHIMIA [82].

\section{Session 12}

Jürgen Stohner, ZHW Winterthur (Fig. 29) was chairman of Session 12, the first session of the last day, introducing Jürgen Gauss, Universität Mainz (Fig. 30), who lectured on 'Rovibrational Spectroscopy and Quantum Chemistry'. He demonstrated the fruitful interplay between high-level quantum-chemical calculations and experimental rovibrational spectroscopic investigations. Spectroscopic parameters (e.g. rotational constants, equilibrium structure and rotation-vibration interaction constants) from high-level quantum chemical calculations are an extremely helpful starting point for the analysis of complex experimental rovibrational spectra. He explained that within a perturbational approach to the spectroscopic problem modern analytic derivative techniques for general coupled cluster models in combination with large basis sets can provide the required spectroscopic constants in a very efficient way. $\mathrm{He}$ discussed some of the problems that arise when relating the experimental ground state rotational constants to molecular structural parameters. The standard approach for polyatomic molecules is to determine spectroscopic ground state rotational constants $B_{0}$ for several isotopomers of the molecule and to relate these constants to their equilibrium values $B_{e}$ or else determine vibrational state dependent $B_{v}$ and extrapolate these to $B_{0}$, but this becomes an increasingly difficult task for larger molecules and increasing number of degrees of freedom. The difficulties related to the determination of the experimental vibration-rotation interaction

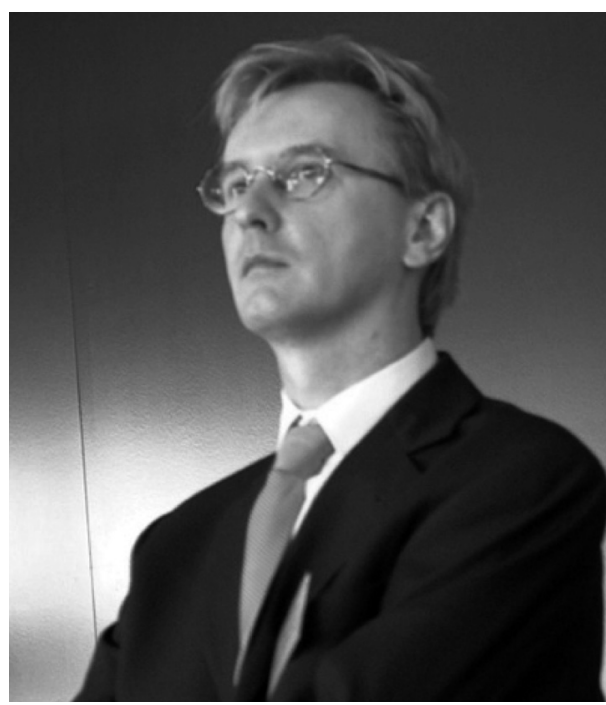

Fig. 29. Jürgen Stohner, Winterthur and Zürich

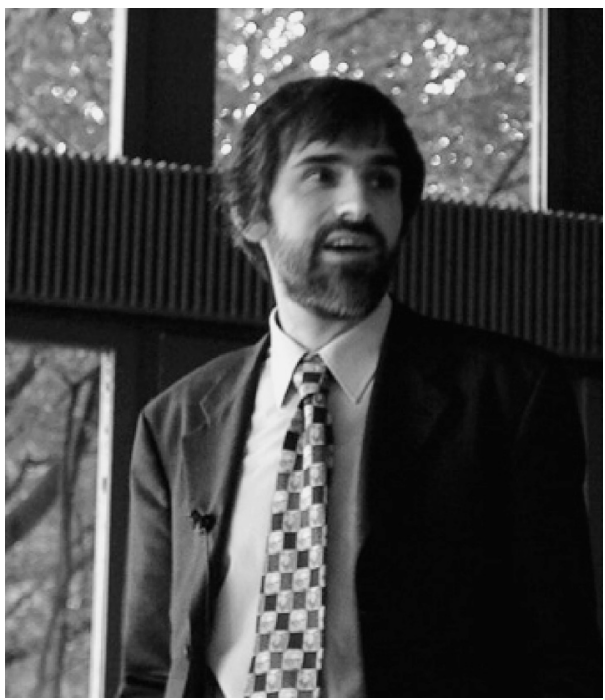

Fig. 30. Jürgen Gauss, Mainz

constants may be overcome by using constants obtained from electronic structure calculations. Gauss showed that the vibration-rotation interaction constants are small compared to the ground state rotational constants and may be calculated with relatively high accuracy. High-quality equilibrium structures may therefore be obtained using experimental rotational constants and calculated vibration-rotation interaction constants [83]. He also reported investigations on the magnitude of various sources of errors in empirical equilibrium structures when the vibration-rotation interaction constants were calculated at various standard $a b$ initio wave function/basis set levels. He stated that empirical equilibrium bond lengths have a relative accuracy of the order of $10^{-3}$ when the vibration-rotation interaction constants were calculated at a correlated level of electronic structure theory. He illustrated this successful interplay of theory and experiment on the examples of $\mathrm{H}_{2} \mathrm{C}_{2}$ and $\mathrm{F}_{-} \mathrm{C}_{4}-\mathrm{H}$. As a most impressive example he reported quantum chemical predictions of the rotational spectrum of $\mathrm{HSOH}$ [84], which led to its infrared and microwave spectroscopic detection in the gas phase. Jürgen Gauss's very stimulating lecture gave rise to considerable discussion among spectroscopists and theoreticians. Some of this expanded on the points already raised in the discussions of the lectures by Olsen, Hirao and Thiel, in particular. Botschwina pointed to some earlier theoretical work on fluoroacetylene [85] and it was pointed out as well that some experimental detection of HSOH had been reported [86] as well as $a b$ initio and mode selective tunneling calculation on various $\mathrm{HSOH}$ isotopomers, including even parity violation in this molecule as part of a larger series of chiral HXYH molecules [87][88].

The following lecture was given by Markus Reiher, Universität Erlangen (Fig. 31) on 'Mode-Tracking: A New Method for 


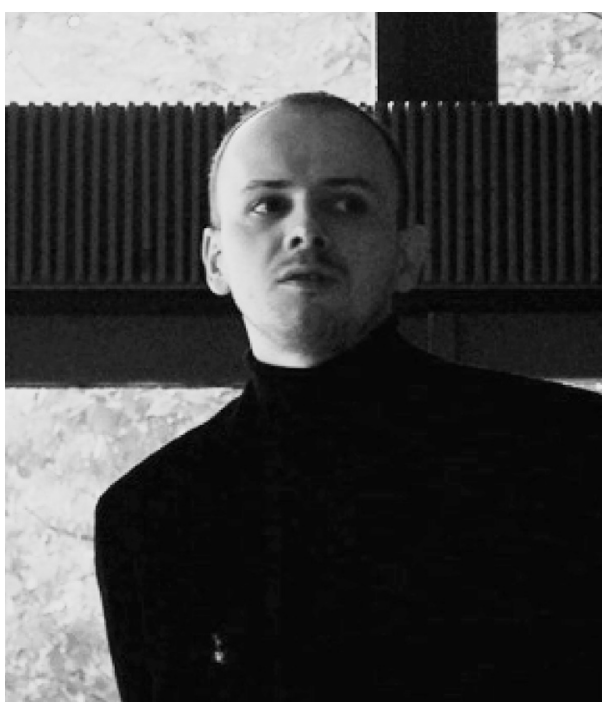

Fig. 31. Markus Reiher, Erlangen

the Calculation of Pre-Selected Molecular Vibrations'. He started his lecture by mentioning that the quantum chemical standard approaches for the calculation of vibrational spectra are usually limited to small molecules. To overcome this problem the socalled mode-tracking approach was developed for the accurate normal mode calculation of a pre-selected molecular vibration, which is important in the experimental spectrum, for instance, in a very large molecule. The vibrational eigenvalue problem is solved in a small subspace of the complete normal coordinate space and one or a couple of predefined modes are projected out of all $3 \mathrm{~N}$ modes of the molecule. He mentioned that this is a great benefit since the calculation of the full Hessian is not necessary and therefore comparatively little computational effort is needed compared to the 'standard' procedure. He discussed some computational details, stating that for a good convergence behavior of the method it is important to generate a sufficiently accurate guess for the desired normal modes and for the preconditioner. This guess should be based on a quantum chemical method, which is computationally less expensive than the method used for the subspace. Therefore one can choose even semiempirical methods. Markus Reiher mentioned that with this approach, it is possible to study relatively large molecules, for example: single-walled carbon nanotubes, large metal complexes or Schmidbaur's cluster [89-91].

\section{Session 13}

Ralph Jaquet (Universität Siegen) chaired Session 13 with two further theoretical highlights. Lorenz S. Cederbaum, Universität Heidelberg (Fig. 32) introduced his contribution with the title 'Intermolecular Decay and Ultrafast Energy Transfer in Clusters and Weakly Bound Systems". He pointed out that with large scale ab initio calculations it is possible to study the relaxation of molecular clusters after excitation by inner-valence ionization. He reported that electronically excited cations relax in general by dissociation and photon emission. Autoionization is forbidden for energetic reasons. The situation changes fundamentally in an inner-valence ionized cluster, which releases its excess energy by emitting an electron. This newly discovered and analyzed process, referred to as Intermolecular Coulombic Decay (ICD) [92-94], is characterized by an inner-valence hole state in a weakly bonded system which can undergo ultrafast relaxation on a femtosecond time scale due to energy transfer to a neighboring atom, followed by electron emission from this neighboring site. He discussed theoretical methods which were developed to pre-

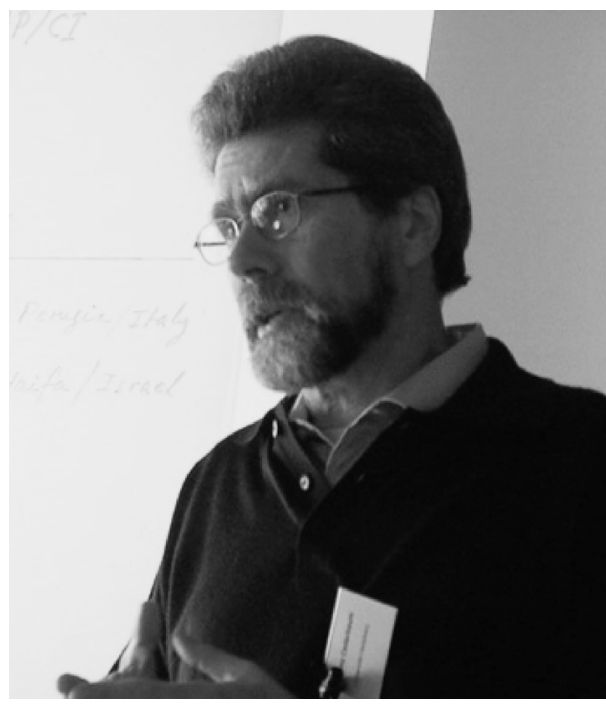

Fig. 32. Lorenz S. Cederbaum, Heidelberg

dict properties (e.g. lifetimes) of molecular systems undergoing electronic decay. These approaches allow the study of the relaxation process of inner-valence holes in clusters. $\mathrm{He}$ noted that for $\mathrm{Ne}$ clusters strong experimental evidence was recently found for an ICD process [95], which was theoretically predicted [92]. He concluded that the underlying ICD process is of a very general nature and it has consequences far beyond clusters and single ionization.

The last morning lecture was given by William H. Miller, University of California/Berkeley (Fig. 33) who spoke about the use of 'Semiclassical Theory to Include Quantum Effects in Classical Molecular Dynamics Simulations'. Bill Miller started his lecture with a summary of theoretical chemical dynamics. Clearly, quantum dynamics is, in principle, required and it is feasible for small molecular systems. It provides state to state reactive scattering transition probabilities and cross sections, time evolution of wave packets and direct quantum calculation of chemical reaction rates, the latter being a major development from Miller himself. Many examples for triatomic systems A + BC, and four atomic systems $\mathrm{AB}+\mathrm{CD}$ exist, the latter constituting about the limit in size of the current state of the art in this field. The second widely used approach is classical molecular dynamics either on empirical potential hypersurfaces ('force fields') or $a b$ initio potential hypersurfaces (such as the density functional theory ab initio molecular dynamics following Car and Parrinello). This approach is feasible for complex systems including proteins and the like, but nuclear dynamics is treated here with 17 th century science. The question is then, how to introduce quantum effects. Basically three strategies are in use (i) Ignore them! (ii) Treat a few degrees of freedom quantum mechanically and the remaining ones classically (iii) Treat all de-

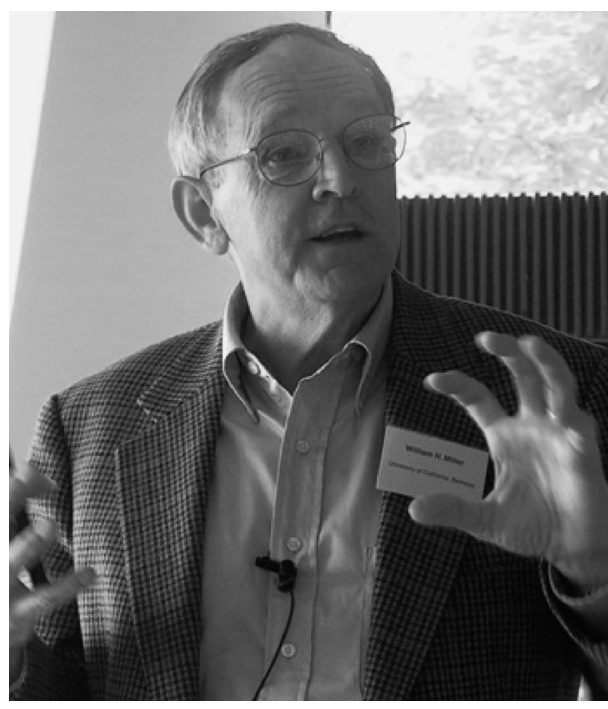

Fig. 33. William H. Miller, Berkeley

grees of freedom semiclassically. It is the third strategy that was further pursued in Miller's lecture. While the basis semiclassical approximation can be found in work of Van Vleck from 1928, the renaissance of this field is largely due to Miller's work about three decades ago [96]. Semiclassical theory provides in general a good description of essentially all quantum effects in molecular dynamics, e.g. interference and coherence, tunneling, vibrational zero point energy, effects of identical particle symmetry or quantization of bounded motion. For small molecules, this has been appreciated and checked by many applications in the past. Miller presented several examples. He concluded that in principle it should be possible to use the semiclassical theory to add in an approximate way quantum effects to classical simulations of chemical reactions in solution, clusters and proteins in complex molecular systems. Quantum effects are added, he explained, by replacing the boundary value problem of semiclassical theory by an average over the initial condi- 
tions of classical trajectories. He then continued with an practical implementation of the semiclassical theory for such complex systems which is based on initial value representations. He reviewed in the last part of his talk the basic ideas of such approaches and described the broad spectrum of applications for a number of chemical problems (for a recent overview see [97]). Among other things Bill Miller's lecture provided an illustration that even in the days of laptops and power point, some of the most brilliant theoretical lectures can be presented using hand-written transparencies.

\section{Session 14}

The final session was chaired by $D o$ minik Marx, University of Bochum (Fig. 34). The first contribution to this session was by Nikos L. Doltsinis (Ruhr-Universität Bochum) with an instructive talk on 'Excited State Tautomerism of the DNA Base Guanine'. He introduced this subject with the fact that although up to six tautomeric forms of guanine coexist in IR-UV spectroscopic molecular beam experiments, only one, the so-called N9H-keto tautomer is usually present in healthy DNA. For both the interpretation of the experimental data and to elucidate the biological relevance of the $\mathrm{N} 9 \mathrm{H}$-keto form his group investigated theoretically the photophysical properties of the guanine tautomers. For such investigations they used a density functional restricted open-shell Kohn-Sham approach [98]. He presented results which show that there are large variations in the extent of structural relaxation in the $S_{1}(\pi \pi *)$ excited state for different guanine tautomers [99]. The keto forms have larger geometric changes upon electronic excitation to the $S_{1}$ state than their enol counterparts. Then he further pointed out that methylation of nitrogen N9 in gua-

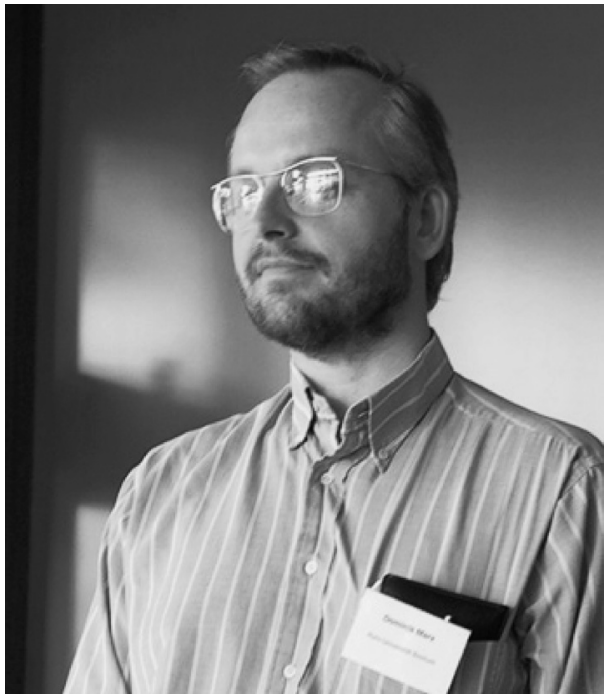

Fig. 34. Dominik Marx, Bochum, chairing the session with Doltsinis and Parrinello nine constitutes an important step towards 'real' biological systems, since this is just in the position of the backbone linkage in DNA. Their current studies suggest that due to heavy structural distortions in the $S_{1}$ state the $\mathrm{S}_{1} \leftarrow \mathrm{S}_{0}$ transition probability of this particular tautomer is rather small. Car-Parrinello calculations [100-102] have demonstrated that large structural changes in $9 \mathrm{Me}-$ keto guanine also result in significantly shorter internal conversion times. Then he summarized their findings for this simple model system and its implications for the photophysical properties of DNA building blocks. He proposed that, apart from a short excited state lifetime, low absorption probability of the isolated nucleobase may contribute to protecting DNA from UV damage. These mechanisms may be exploited in future synthesis of photostable biomolecules.

The final lecture of the symposium was given by Michele Parrinello, ETH Zürich and Swiss Center for Scientific Computing (CSCS), Manno (Fig. 35) entitled 'Calculation of Free Energy Surfaces in Classical and $a b$ initio Molecular Dynamics'. He started his lecture by explaining that at least by now he was moving from Newton to Gibbs (in reply to a comment in Bill Miller's lecture). The free energy surface of complex systems is usually characterized by the presence of deep minima separated often by large barriers. The transitions over such barriers correspond to chemical reactions and conformational changes. He explained that the direct simulation of these processes is often not possible because of the exponential dependence of the rates on the barrier height (this means that the free energy minimum near the start configuration will eventually not be left). This results in a severe time scale problem. Then he introduced his 'metadynamics' method for exploration of the properties of the multidimensional free energy surfaces of complex

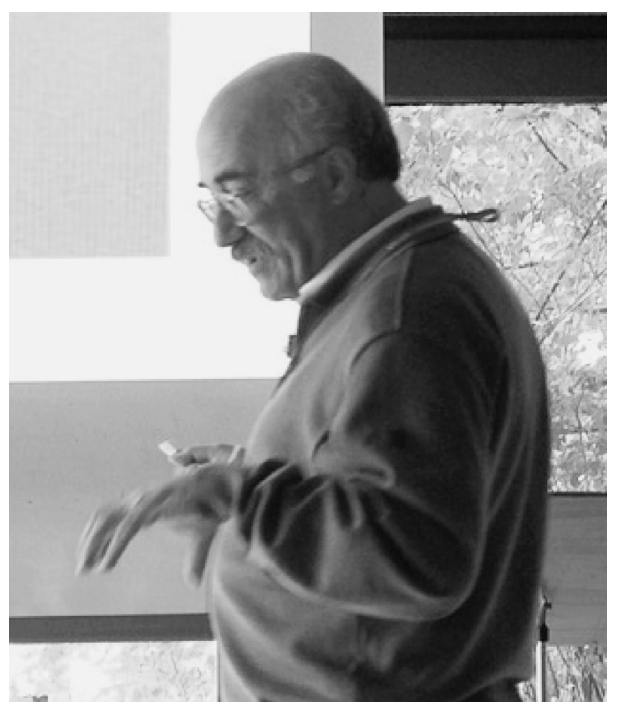

Fig. 35. Michele Parrinello, Lugano and Zürich many-body systems by means of a coarsegrained non-Markovian dynamics in a space defined by a few collective coordinates. He explained that the characteristic feature of this dynamics is the presence of a 'history'-dependent potential term that, in time, fills the minima in the free energy surface. This allows a very efficient exploration of the free energy surface as a function of the chosen collective coordinates. Then he presented as a demonstration of the power of this approach some impressive examples including simulations of some fundamental real-life chemistry such as $\mathrm{Na}^{+}$and $\mathrm{Cl}^{-}$in water or $\mathrm{NO}_{3}{ }^{-}$ reduction to $\mathrm{NH}_{3}$ by pyrite, which takes place in oceanic hydrothermal vents and may be important in early evolution. Some of the work reported in this lecture is published in [103-105].

\section{Posters}

The posters of this meeting were numerous and reflected upon the areas treated in the lectures, including however also further areas of research. The poster sessions led to very lively scientific discussions, although some participants noted a certain lack of beer that might have increased the amount of discussions (but not necessarily their quality... ). It is impossible here to give a fair survey of the very wide range of results covered in the poster sessions. We shall select here only one area related to some current research interests of the organizers. Given the fact that the hosts of the meeting withheld their work from the oral presentations, following a good tradition to leave more room for the lectures of the guests, this particular selection may perhaps seems fair enough. After the discussions on 17th century science, 19 th century science and early 20th century science (i.e. quantum chemistry being based on the Schrödinger and Dirac equations of 1926-1929 or semiclassical theory being based on the even earlier 'old quantum theory') this area of research from the posters leads into the late 20 th century and 21 st century with electroweak theory and the standard model and beyond.

The topic to be addressed here can be summarized under the relatively newly coined term 'electroweak quantum chemistry' [106][107]. It deals with the inclusion of the parity violating weak nuclear force in quantum chemical calculations, with the aim of properly treating the quantum dynamics and spectroscopy of chiral molecules including parity violation as reviewed in [108][109]. About five posters dealt with this intriguing topic, which led to considerable discussions. Some of the work by now is available in print [68][87][88] [106-108][110-112]. 


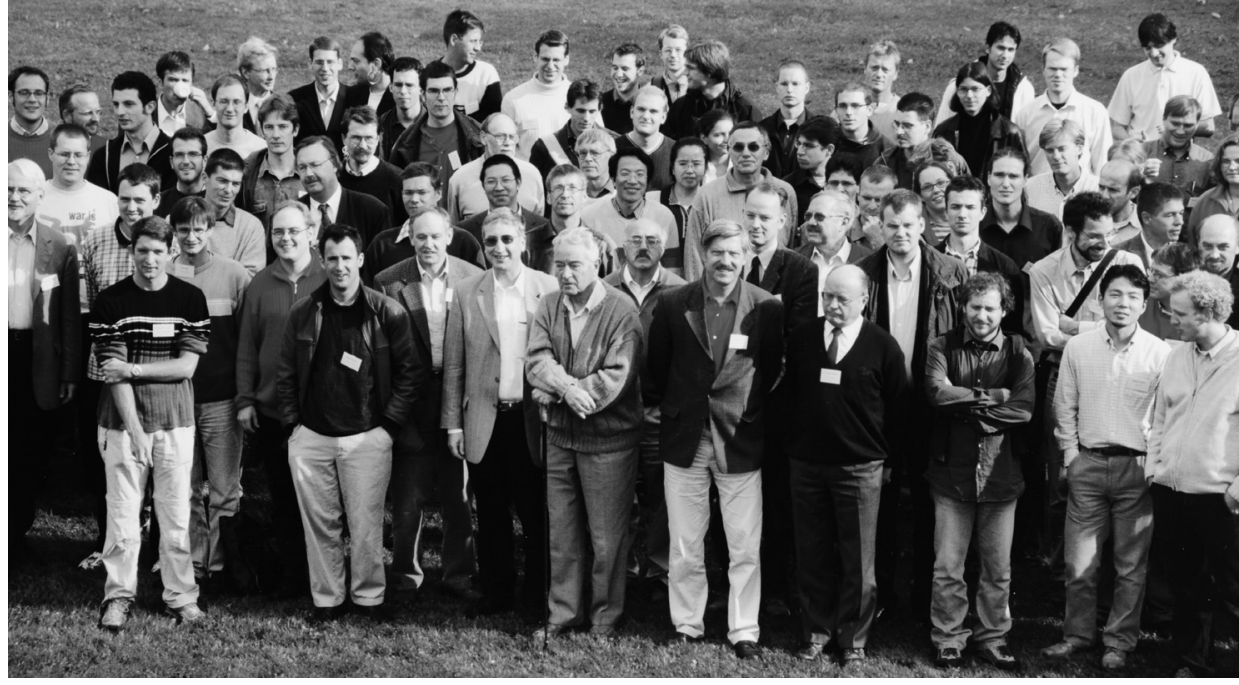

Fig. 36. This partial group picture presents the central section of the conference photo and includes some chairmen and speakers mentioned in the text but missing in the picture series. In the front row in the middle one finds with sunglasses the conference chairman Martin Quack, to the left of him, diagonally towards the back Bill Miller (Berkeley) and Ralph Jacquet (with tie, Siegen). To the right from Martin Quack one finds in the front row Tino Gäumann (Lausanne), Peter Botschwina (Göttingen), W.H. Eugen Schwarz (Siegen), Fabio Mariotti (Mülheim and Zürich). Towards the back the second person behind Fabio Mariotti is Nikos Doltsinis, whereas in the diagonal to the right from there one sees Martin Willeke. Some other known faces can be identified by comparison with the previous figures.

\section{Extracurricular Activities}

The report would be incomplete without mentioning at least one of the extracurricular activities. Splendid weather on Tuesday afternoon made the excursion to the Stockhorn an exceptional event, with wide views over the Bernese alps. Several options were available including one all bus and cable car option, but also quite extensive and strenuous hiking, which some of the participants chose, while the large majority put up with a combined hiking and cable car version. Fig. 36 shows the picture of some part of the group (after recovering from the excursion) and Fig. 37 shows the mountain and lake theme of the conference logo which accompanied the excursion.

\section{Acknowledgment}

Particular support was provided by the division chemical research of the Swiss Chemical
Society. Further financial support came from the ETH Zürich (SEP - Computational Sciences), the laboratory of Physical Chemistry of ETH, the Royal Society of Chemistry (for the PCCP lecture), from the Swiss National Science Foundation, and Swiss chemical industry, the KGF (see Fig. 38). Most of the photographs were taken by Sofia Deloudi, while the group photo was taken by Marius Lewerenz, thanks to all of them for their expert help and great work. For two minireviews see [113][114]

Received: April 8, 2004

[1] Proceedings of the 39th Symposium on Theoretical Chemistry, 'Molecular Spectroscopy and Dynamics', 28 September to 2 October 2003, Eds. J. Stohner, M. Quack, Gwatt, Switzerland (2003).

[2] M. Quack, W. Kutzelnigg, 'Molecular Spectroscopy and Molecular Dynamics: Theory and Experiment', Ber. Bunsenges. Phys. Chem. 1995, 99, 231-245 review; Ber. Bunsenges. Phys. Chem. 1995, 99, 246-582 papers.
[3] M. Quack, J. Troe, Ber. Bunsenges. Phys. Chem. 1974, 78, 240.

[4] M. Quack, J. Troe, 'Statistical Methods in Scattering', 'Theoretical Chemistry: Advances and Perspectives', 1981, 6B, 199.

[5] M. Quack, J. Troe, 'Statistical Adiabatic Channel Model', in Encyclopedia of Computational Chemistry, Vol. 4, Ragué Schleyer, Eds. P. von Ragué Schleyer, N. Allinger, T. Clark, J. Gasteiger, P.A. Kollman, H.F. Schaefer III, P.R. Schreiner, Wiley 1998, p. 2708.

[6] A.I. Maergoiz, E.E. Nikitin, J. Troe, V.G Ushakov, J. Chem. Phys. 1996, 105, 6263

[7] A.I. Maergoiz, E.E. Nikitin, J. Troe, V.G. Ushakov, J. Chem. Phys. 1998, 108, 5265; A.I. Maergoiz, E.E. Nikitin, J. Troe, V.G. Ushakov, J. Chem. Phys. 1998, 108, 9987.

[8] A.I. Maergoiz, E.E. Nikitin, J. Troe, V.G. Ushakov, J. Chem. Phys. 2002, 117, 4201.

[9] E.I. Dashevskaya, A.I. Maergoiz, J. Troe, I. Litvin, E.E. Nikitin, J. Chem. Phys. 2003 , $118,7313$.

[10] B. Kuhn, T.R. Rizzo, D. Luckhaus, M. Quack, M. Suhm, J. Chem. Phys. 1999, 111, 2565.

[11] V. Yakhontov, M. Jungen, Can. J. Phys. 2002, 80, 1432

[12] W. Klopper, J. Noga, ChemPhysChem 2003, 4,32 .

[13] W. Klopper, C.C.M. Samson, J.Chem. Phys. 2002, 116, 6397.

[14] C.C.M. Samson, W. Klopper, T. Helgaker, Comp. Phys. Chem. 2002, 149, 1.

[15] D. Luckhaus, J. Chem. Phys. 2000, 113, 1329.

[16] D. Luckhaus, J. Chem. Phys. 2003, 118, 8797.

[17] D. Luckhaus, Chimia 2002, 56, 8; Werner Prize Lecture.

[18] M. Hippler, M. Quack, Chem. Phys. Lett. 1999, 314, 273

[19] M. Hippler, M. Quack, J. Chem. Phys. 2002, 116, 6045.

[20] P. Birza, T. Motylewski, D. Khoroshev, A. Chirokolava, H. Linnartz, J.P. Maier, Chem. Phys. 2002, 283, 119.

[21] H. Ding, T.W. Schmidt, T. Pino, A.E. Boguslavskiy, F. Güthe, J.P. Maier, J. Chem. Phys. 2003, 119, 814.

[22] H. Ding, T.W. Schmidt, T. Pino, F. Güthe, J.P. Maier, Phys. Chem. Chem. Phys. 2003 , 5,4772 .

[23] A. Wüest, F. Merkt, J. Chem. Phys. 2004, 120,638

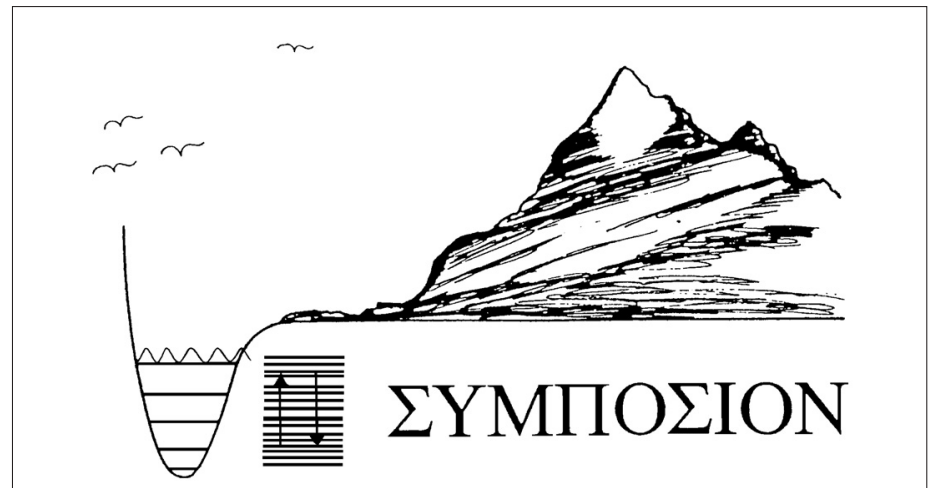

Fig. 37. Mountain and lake (and some molecular spectroscopy and dynamics and more) encoded in the conference logo

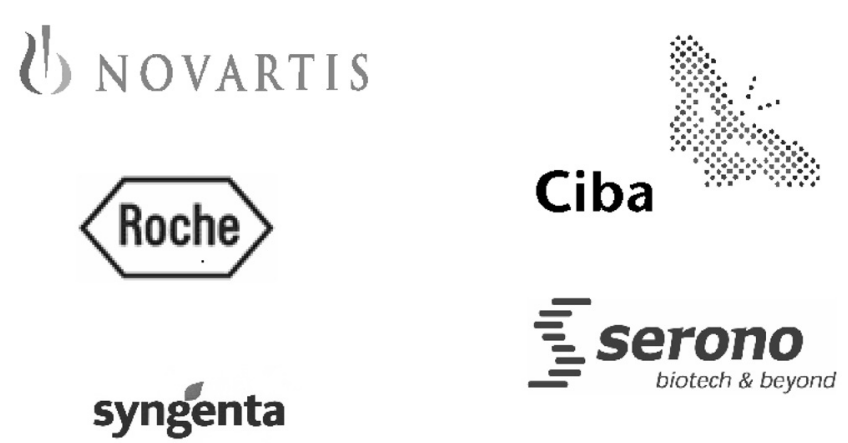

Fig. 38. Industry Sponsors 
[24] H.J. Wörner, U. Hollenstein, F. Merkt, Phys. Rev. 2003, A68, 032510.

[25] S. Willitsch, A. Wüest, F. Merkt, Chimia 2004, 58, 281.

[26] H.-J. Werner, F.R. Manby, P.J. Knowles, J. Chem. Phys. 2003, 118, 8149.

[27] M. Schütz, F.R. Manby, Phys. Chem. Chem. Phys. 2003, 5, 3349.

[28] T.A. Ruden, T. Helgaker, P. Jorgensen, J. Olsen, Chem. Phys. Lett. 2003, 371, 62.

[29] F. Pawlowski, P. Jorgensen, J. Olsen, F. Hegelund, T. Helgaker, K.L. Bak, J.F. Stanton, J. Chem. Phys. 2002, 116, 6482.

[30] M. Lewerenz, M. Quack, J. Chem. Phys. 1988, 88, 5408 .

[31] M. Quack, M. Suhm, J. Chem. Phys. 1991, 95, 28.

[32] W. Klopper, M. Quack, M. Suhm, J. Chem. Phys. 1998, 108, 10096

[33] H. Hollenstein, R. Marquardt, M. Quack, M. Suhm, J. Chem. Phys. 1994, 101, 3588.

[34] M. Quack, Annu. Rev. Phys. Chem. 1990, 41, 839.

[35] A. Beil, H. Hollenstein, O. Monti, J. Stohner, M. Quack, J. Chem. Phys. 2000, 113, 2701.

[36] J. Pochert, M. Quack, J. Stohner, M. Willeke, J. Chem. Phys. 2000, 113, 2719.

[37] T. Ziegler, A. Rauk, Theor. Chim. Acta 1977, $46,1$.

[38] K. Kitaura, K. Morokuma, Int. J. Quantum Chem. 1976, 10, 325.

[39] M. Lein, A. Szabó, A. Kovács,G. Frenking, Faraday Discuss. 2003, 124, 365.

[40] M.S. Nechaev, V.M. Rayón, G. Frenking, $J$. Phys. Chem. A 2004, 108, 3134.

[41] S. Hirata, T. Yanai, W.A. de Jong, T. Nakajima, K. Hirao, J. Chem. Phys. 2004, 120, 3297.

[42] Y. Kurashige, H. Nakano, K. Hirao, J. Phys. Chem. A 2004 10.1021/jp031133k Web Release.

[43] G. Kresse, VASP - Vienna ab initio simulation package, homepage at http://cms.mpi. univie.ca.at/vasp.

[44] G. Kresse, A. Gil, P. Sautet, Phys. Rev. 2003, B68, art. no. 073401-1.

[45] A. Gil, A. Clotet, J.M. Ricart, G. Kresse, M Garcia-Hernandez, N. Rösch, P. Sautet, Surf. Science 2003, 530, 71.

[46] R.D. Beck, T.R. Rizzo, Chimia 2004, 58, 306.

[47] R.D. Beck, P. Maroni, D.C. Papageorgopoulos, T.T. Dang, M.P. Schmid, T.R. Rizzo, Science 2003, 302, 98.

[48] R. Marquardt, M. Quack, I. Thanopulos, D. Luckhaus, J. Chem. Phys. 2003, 118, 643.

[49] R. Marquardt, M. Quack, I. Thanopulos, D. Luckhaus, J. Chem. Phys. 2003, 119, 10724.

[50] F. Cuvelier, S. Hervé, R. Marquardt, K. Sagui, Chimia 2004, 58, 296.

[51] M. Quack, 'Molecular femtosecond quantum dynamics between less than yoctoseconds and more than days: Experiment and theory', Chapter 27, p. 781-818, in Femtosecond Chemistry, Eds. J. Manz, L. Woeste, Verlag Chemie, Weinheim, 1995.

[52] R. de Vivie-Riedle, V. De Waele, L. Kurtz, E. Riedle, J. Phys. Chem. A 2003, 107, 10591.

[53] T. Hornung R. de Vivie-Riedle, Europhys. Lett. 2003, 64, 703.

[54] D. Geppert, A. Hofmann, R. de VivieRiedle, J. Chem. Phys. 2003, 119, 5901.
[55] R. Marquardt, M. Quack, Z. Physik D 1996, 36, 229.

[56] C. Tanner, C. Manca, S. Leutwyler, Science 2003, 302, 1736

[57] A. Bach, C. Tanner, C. Manca, H. Frey, S. Leutwyler, J. Chem. Phys. 2003, 119, 5933.

[58] C. Manca, C. Tanner, S. Leutwyler, Chimia 2004, 58, 287.

[59] F. Madeja, M. Havenith, J. Chem. Phys. 2002, 117, 7162 .

[60] M. Quack, S. Jans-Bürli, 'Molekulare Thermodynamik und Kinetik', Teil 1 Chemische Reaktionskinetik, $V d F$ Publishers, Zürich, 1986 (see A-62 therein).

[61] J. Blumberger, T.K. Ha, J. Paff, M. Quack, G. Seyfang, 'Time resolved FTIR-detection of IR-multiphoton initiated dissociation of formic acid dimers: Evidence for a dimer with a single strong hydrogen bond', in SASP 2000, Proc. 12th Symp. on Atomic and Surface Physics and Related Topics, Eds. D. Bassi, P. Tosi, Costa di Folgaria (Trento) 2000, page PB-2.

[62] M. Goubet, B. Madebène, M. Lewerenz, Chimia 2004, 58, 291

[63] C. Kötting, W. Sander, J. Breidung, W. Thiel, M. Senzlober, H. Bürger, J. Am. Chem. Soc. 1998, 120, 219.

[64] H. Lin, W. Thiel, S.N. Yurchenko, M. Carvajal, P. Jensen, J. Chem. Phys. 2002, 117 11265.

[65] F. Mariotti, R. Marquardt, M. Quack, W. Thiel, 'On an accurate global potential hypersurface of $\mathrm{NH}_{3}$ ', (work in progess).

[66] W. Thiel, Chimia 2004, 58, 276.

[67] N. Borho, M.A. Suhm, Phys. Chem. Chem. Phys. 2002, 4, 2721.

[68] R. Berger, M. Quack, G. Tschumper, Helv. Chim. Acta 2000, 83, 1919.

[69] N. Borho, M.A. Suhm, Org. Biomol. Chem. 2003, 1,4351

[70] D. Luckhaus, M. Quack, U. Schmitt, M.A. Suhm, Ber. Bunsenges. Phys. Chem. 1995, 99, 457.

[71] T. Häber, U. Schmitt, C. Emmeluth, M. Suhm, Faraday Discuss. Chem. Soc. 2001, 118, 331 .

[72] S. Portmann, A. Inauen, H.P. Lüthi, S Leutwyler, J. Chem. Phys. 2000, 113, 9577.

[73] N. Borho, T. Häber, M.A. Suhm, Phys.Chem.Chem.Phys. 2001, 3, 1945.

[74] T.A. Wesolowski, A. Warshel, J. Phys. Chem. 1993, 97, 8050.

[75] T.A. Wesolowski, F. Tran, J. Chem. Phys. 2003, 118, 2072

[76] T.A. Wesolowski, Chimia 2004, 58, 311.

[77] V. Ganduglia-Pirovano, J. Sauer, Phys. Rev. B, 2004, submitted.

[78] V. Brazdova, V. Ganduglia-Pirovano, J. Sauer, Phys. Rev. B 2004, in press.

[79] D. Schröder, J. Loos, M. Engeser, H. Schwarz, H.-C. Jankowiak, R. Berger, R. Thissen, O. Dutuit, J. Döbler, J. Sauer, Inorg. Chem. 2004, 43, 1976.

[80] J. Döbler, M. Pritzsche, J. Sauer, 2004, unpublished.

[81] O. Hübner, J. Sauer, Phys. Chem. Chem. Phys. 2002, 4, 5234.

[82] C.A. Daul, Chimia 2004, 58, 316.

[83] J. Gauss, J.F. Stanton, J. Phys. Chem. A $2000,104,2865$
[84] G. Winnewisser, F. Lewen, S. Thorwirth, M. Behnke, J. Hahn, J. Gauss, E. Herbst, Chemistry 2003, 9, 5501 .

[85] P. Botschwina, M. Oswald, J. Fluegge, A. Heyl, R. Oswald, Chem. Phys. Lett. 1993, 209, 117.

[86] M. Iraqi, H. Schwarz, Chem. Phys. Lett. 1994, 221, 359.

[87] M. Quack, M. Willeke, Helv. Chim. Acta 2003, 86, 1641

[88] M. Gottselig, M. Quack, J. Stohner, M. Willeke, Int. J. Mass Spec. 2004, 233, 373.

[89] J. Neugebauer, M. Reiher, C. Kind, B. A. Hess, J. Comp. Chem. 2002, 23, 895.

[90] M. Reiher, J. Neugebauer, B. A. Hess, Z. Phys. Chem. 2003, 217, 91.

[91] M. Reiher, J. Neugebauer,J. Chem. Phys. 2003, 118, 1634

[92] L.S. Cederbaum, J. Zobeley, F. Tarantelli, Phys. Rev. Lett. 1997, 79, 4778.

[93] R. Santra, J. Zobeley, L.S. Cederbaum, N. Moiseyev, Phys. Rev. Lett. 2000, 85, 4490.

[94] R. Santra, J. Zobeley, L.S. Cederbaum, Phys. Rev. Lett. B 2001, 64, art. no. 245104.

[95] S. Marburger, O. Kugeler, U. Hergenhahn, T. Möller, Phys. Rev. Lett. 2003, 90, art. no. 203401.

[96] W.H. Miller, Adv. Chem. Phys. 1975, 30 77.

[97] W.H. Miller, J. Phys. Chem. A 2001, 105, 2942.

[98] I. Frank, J. Hutter, D. Marx, M. Parrinello, J. Chem. Phys. 1998, 108, 4060.

[99] H. Langer, N. L. Doltsinis, J. Chem. Phys. 2003, 118, 5400.

[100] N.L. Doltsinis, D. Marx, Phys. Rev. Lett. 2002, 88, art. no. 166402.

[101] N.L. Doltsinis, D. Marx, J. Theor. Comp. Chem. 2002, 1, 319.

[102] N.L. Doltsinis in 'Quantum Simulations of Complex Many-Body Systems: From Theory to Algorithms', Eds. J. Grotendorst, D. Marx, A. Muramatsu (NIC, FZ Jülich, 2002), for downloads see http://www.fzjuelich.de/nic-series/volume10/doltsinis.pdf.

[103] A. Laio, M. Parrinello, Proc. Natl. Acad. Sci. 2002, 99, 12562.

[104] R. Martonak, A. Laio, M. Parrinello, Phys. Rev. Lett. 2003, 90, art. no. 075503.

[105] M. Iannuzzi, A. Laio, M. Parrinello, Phys. Rev. Lett. 2003, 90, art. no. 238302.

[106] A. Bakasov, T.K. Ha, M. Quack, J. Chem. Phys. 1998, 109, 7263.

[107] R. Berger, M. Quack, J. Chem. Phys. 2000, 112,3148 .

[108] M. Quack, J. Stohner, J. Chem. Phys. 2003 , $119,11228$.

[109] M. Quack, Angew. Chem. Intl. Ed. 2002, 41, 4618.

[110] G. Laubender, R. Berger, ChemPhysChem 2003, 4, 395 .

[111] R. Berger, M. Quack, A. Sieben, M. Willeke, Helv. Chim. Acta 2003, 86, 4048.

[112] M. Gottselig, M. Quack, M. Willeke, Isr. J. Chem. 2004, in press.

[113] M. Mladenovic, S. Schmatz, Nachr. Chem. 2004, 62, 67 .

[114] S. Schmatz, M. Mladenovic, Bunsen Magazin, 2004, 6, 16. 